\title{
The Thermodynamic Formalism for Expanding Maps ${ }^{\star}$
}

\author{
David Ruelle \\ I.H.E.S., F-91440 Bures-sur-Yvette, France
}

\begin{abstract}
Let $f: X \mapsto X$ be an expanding map of a compact space (small distances are increased by a factor $>1)$. A generating function $\zeta(z)$ is defined which counts $f$-periodic points with a weight. One can express $\zeta$ in terms of nonstandard "Fredholm determinants" of certain "transfer operators", which can be studied by methods borrowed from statistical mechanics. In this paper we review the spectral properties of the transfer operators and the corresponding analytic properties of $\zeta(z)$. Gibbs distributions and applications to Julia sets are also discussed. Some new results are proved, and some natural conjectures are proposed.
\end{abstract}

\section{Introduction}

The thermodynamic formalism is a body of ideas and results originating in equilibrium statistical mechanics and which has had a considerable impact on the study of the ergodic theory of hyperbolic differentiable dynamical systems. ${ }^{1}$ The easiest case, for which the strongest results are known, is that of expanding maps. Of particular interest are the zeta functions associated with such maps (see Ruelle $[18,19]$ and their correlation functions and corresponding resonances and Gibbs distributions (see Ruelle [22]). Important ideas for the analysis of these concepts have been introduced by Fried [8], Tangerman [27], Pollicott [15], Haydn [10].

The purpose of the present paper is to review the thermodynamic formalism (or at least part of it) for expanding maps, with some extensions and some new proofs. We shall discuss in detail the spectrum of the transfer operators and describe conjectures and known results about the analyticity of the zeta functions. The theory developed for expanding maps has applications to the thermodynamic formalism of Axiom A diffeomorphisms and flows, and in particular to their zeta

\footnotetext{
* This is an expanded version of the Bowen lectures given by the author at U.C. Berkeley in November 1988

1 For the origins of the subject, see in particular Sinai [24, 25, 26]; Ruelle [17]; Bowen and Ruelle [5], and the monographs by Bowen [3] and Ruelle [20]
} 
functions (see Parry and Pollicott [13], Fried [8]), but we shall not discuss these questions here. [Note added in proof: Conjectures $A$ and $B$ below have now been proved, see D. Ruelle, An Extension of the theory of Fredholm determinants, preprint.]

\section{Assumptions}

In what follows we shall use $C^{r}$ functions, where $\mathbf{r}$ is an integer $r \geqq 1$, or $\mathbf{r}=(r, \alpha)$ with integer $r \geqq 0$ and $0<\alpha \leqq 1$. [We say that a function is $C^{(r, \alpha)}$ if it is $C^{r}$ and if the $r^{\text {th }}$ derivative is Hölder continuous of exponent $\left.\alpha\right]$. We write $|\mathbf{r}|=r$ in the first case, $|\mathbf{r}|=r+\alpha$ in the second case, and say that $\mathbf{r}$ is finite. We shall also use $C^{\infty}$ functions and $C^{\omega}$ functions (i.e., real analytic functions) and write $|\infty|=|\omega|=\infty$.

Let a $C^{\infty}\left(\right.$ or $\left.C^{\omega}\right)$ manifold $M$ be given, with $\operatorname{dim} M<\infty$, and a Riemann metric d. Also let $X \subset U \subset M$, where $X$ is compact and $U$ open. We assume that a continuous map $f: U \mapsto M$ is given such that

(a) There are $\varepsilon>0$ and $\theta \in(0,1)$ such that

$$
d(f x, f y) \geqq \theta^{-1} d(x, y)
$$

whenever $x, y \in U$ and $d(x, y)<\varepsilon$.

(b) $X=\left\{x \in U: f^{n} x \in U\right.$ for all $\left.n>0\right\}$.

(c) If $\mathcal{O}$ is a nonempty open subset of $X$ (for the topology induced by $U$ ) there is $n>0$ such that $f^{n} \mathcal{O}=X$.

(d) $f$ is $C^{\mathbf{r}}$.

Condition (a) expresses that $f$ is expanding, (b) and (c) imply the invariance property $f X=X,(\mathrm{c})$ expresses that $f$ is topologically mixing. [It is convenient to assume topological mixing. More generally, one could decompose the nonwandering set into topologically transitive pieces, and then reduce to mixing maps, see [20] Chap. 7]. If $\mathbf{r}=(0, \alpha)$, condition (d) is not needed, it suffices to take $f$ continuous.

The above setup applies to the case of an expanding map of a compact manifolds, ${ }^{2}$ and also to the case of a hyperbolic Julia set $X$ when $f$ is a rational map of the Riemann sphere $M^{3}$

1.1 Remark. If $\mathbf{r}=(0, \alpha)$, we can do without the manifold $M: X$ is a compact set with a metric $d$, and a continuous map $f: X \mapsto X$ is given such that (a) and (c) hold. The conditions (b) and (d) are not needed, but we have to assume the existence of local inverses as expressed by Lemma 1.3 below.

Returning to general $\mathbf{r}$ we note that we may take for $U$ an arbitrarily small neighborhood of $X$; we can in particular assume that $f U \supset$ closure $U$ as the following result indicates.

1.2 Lemma. There is an open set $\tilde{U}$ such that $X \subset \tilde{U} \subset U$ and $f \tilde{U} \supset$ closure $\tilde{U}$.

Choose $\delta$ such that $0<\delta<\varepsilon$ and $U$ contains the $\delta$-neighborhood of $X$. Take $x$ with dense orbit in $X$, and choose $n$ such that $\left\{f^{k} x: 0 \leqq k<n\right\}$ is $\delta$-dense in $X$ and

\footnotetext{
${ }^{2}$ Here $X=U=M$, see [7] for a characterization of such maps.

${ }^{3}$ See [21]
} 
$d\left(f^{n} x, x\right)<\left(\theta^{-1}-1\right) \delta$. The lemma is verified if $\tilde{U}$ is the union of the open balls $B_{k}(\delta)$ of radius $\delta$ centered at $f^{k} x$ for $k=0, \ldots, n-1$. [Because of (a) and Brouwer's theorem on the "invariance of domain", we have $f B_{k}(\delta) \supset B_{k+1}\left(\theta^{-1} \delta\right)$.]

As we have just seen, if $x \in X$, the ball $B_{f x}\left(\theta^{-1} \delta\right)$ of center $f x$ and radius $\theta^{-1} \delta$ is contained in the image $f B_{x}(\delta)$. Therefore, $f$ has a unique local inverse $\psi: B_{f x}\left(\theta^{-1} \delta\right) \mapsto B_{x}(\delta)$. [Furthermore, since $\psi$ is contracting, this map is Lipschitz, i.e., of class $C^{(0,1)}$. This is why (d) will not be needed if $r=(0, \alpha)$. If $f$ is differentiable, (d) yields that $\psi$ is $\left.C^{\mathrm{r}}\right]$.

1.3 Lemma. If $x, y^{\prime} \in X$ and $d\left(f x, y^{\prime}\right)<\delta$, there is a unique $y \in X$ such that $f y=y^{\prime}$ and $d(x, y)<\delta$. Furthermore $d(x, y) \leqq \theta d(f x, f y)$.

This results from the existence of the local inverse $\psi$.

1.4 Lemma. Let $\mathbf{I}$ denote the set of $f$-invariant probability measures on $X$, equipped with the vague (=weak) topology, and let $h(\rho)=h_{g}(\rho)$ be the entropy of $\rho \in \mathbf{I}$.

If $g: X \mapsto \mathbb{R}$ is continuous and $g \geqq 0$, the function

$$
\rho \mapsto h(\rho)+\int \rho(d x) \log g(x)
$$

is upper semi-continuous on $\mathbf{I}$. We define the pressure of $\log g$ by

$$
P(\log g)=\max _{\rho \in \mathbf{I}}(h(\rho)+\rho(\log g)) .
$$

If $\rho \in \mathbf{I}$ realizes the maximum, $\rho$ is called an equilibrium state. For Hölder continuous $g>0$, there is a unique equilibrium state.

Since $f$ is expanding, $h$ is upper semi-continuous (Bowen [13]). To show that $\rho \mapsto \rho(\log g)$ is upper semi-continuous, let us assume that $\rho_{n} \rightarrow \rho$ and that $\rho_{n}(\log g) \rightarrow a \neq-\infty$. For $N>0$, let $S=\{x \in X: \log g>-N\}$. We have

$$
\int_{S} \rho_{n}(d x) \log g(x) \geqq \rho_{n}(\log g) \rightarrow a,
$$

hence

$$
\int_{S} \rho(d x) \log g(x) \geqq \lim _{n \rightarrow \infty} \int_{S} \rho_{n}(d x) \log g(x) \geqq a
$$

and

$$
\rho(\log g)=\lim _{N \rightarrow \infty} \int_{S} \rho(d x) \log g(x) \geqq a .
$$

[Note that $\rho\{x: g(x)=0\}=0$ since $\rho=$ vague $\lim \rho_{n}$ and $\rho_{n}(\log g) \rightarrow a \neq-\infty$.]

The uniqueness of equilibrium state for Hölder continuous $g>0$ follows from symbolic dynamics (see below), and is a standard result, for which see [20] Chap. 7.

\section{Symbolic Dynamics}

2.1 Proposition (Markov partitions). For any $\delta>0$ we may write $X$ as a finite union $X=\bigcup_{i \in \mathbf{I}} X_{i}$ of closed nonempty sets $X_{i}$ with diam $X_{i}<\delta$. Furthermore, with the topology of $X$ induced by $M$, 
(i) int $X_{i}$ is dense in $X_{i}$,

(ii) int $X_{i} \cap$ int $X_{j}=\varnothing$ if $i \neq j$,

(iii) each $f X_{i}$ is a union of sets $X_{j}$.

Define

$$
t_{i j}=\left\{\begin{array}{ll}
1 & \text { if } f X_{i} \supset X_{j} \\
0 & \text { otherwise }
\end{array},\right.
$$

then some power $t^{N}$ of the $I \times I$ matrix $\left(t_{i j}\right)$ has all its entries strictly positive.

Markov partitions have been introduced by Sinai $[24,25]$, and generalized by Bowen [2]. Here, the existence of a Markov partition follows from the fact that we have a compact metric space $X$ with a continuous map $f$ of $X$ onto itself satisfying Lemma 1.3. [This is proved in [20], Chap. 7.] The fact that $t^{N}$ has strictly positive entries for some $N$ reflects the assumed topological mixing of $f$.

2.2 Proposition (Symbolic dynamics). We assume that $\delta$ has been chosen small enough. Let

$$
\Omega=\left\{(\xi(n))_{n \geqq 0}: \xi(n) \in I \text { and } t_{\xi(n) \xi(n+1)}=1\right\}
$$

and define

$$
\tau(\xi(n))_{n \geqq 0}=(\xi(n+1))_{n \geqq 0} .
$$

If $I$ is given the discrete topology, $\Omega$ is a compact subset of the product $I^{\mathbb{N}}$, and the shift $\tau: \Omega \mapsto \Omega$ is continuous. When $\xi=(\xi(n)) \in \Omega$, the intersection $\bigcap_{n \geqq 0} f^{-n} X_{\xi(n)}$ consists
of a single point $\pi(\xi)$. Furthermore

(i) The map $\pi: \Omega \mapsto X$ is continuous onto,

(ii) $\pi \tau=f \pi$,

(iii) $\pi^{-1}$ is uniquely defined on the residual set,

$$
X \backslash \bigcup_{n \geqq 0} f^{n} \bigcup_{i \in I}\left(X_{i} \backslash \operatorname{int} X_{i}\right)
$$

(iv) The number card $\left(\pi^{-1} x\right)$ is bounded on $X$,

(v) $\tau$ is topologically mixing.

Symbolic dynamics consists in replacing the study of the dynamical system $(X, f)$ by that of $(\Omega, \tau)$. This is the reason why Markov partitions were introduced by Sinai. For the specific results given above, see Bowen $[2,3,4]$, or [20] Chap. 7.

A dynamical system $(\Omega, \tau)$, where $\Omega$ and $\tau$ are constructed as in (2.1), (2.2) from a finite set $I$ and a matrix $\left(t_{i j}\right)$ with entries 0,1 , is called a subshift of finite type.

2.3 Proposition. Given $\delta>0$, one can choose open sets $U_{i} \subset U$ such that $X_{i} \subset U_{i}$, $\operatorname{diam} U_{i}<\delta, X_{i_{0}} \cap X_{i_{1}} \cap \cdots \cap X_{i_{k}}=\varnothing$ implies $U_{i_{0}} \cap U_{i_{1}} \cap \cdots \cap U_{i_{k}}=\varnothing$, and $f U_{i} \supset$ closure $U_{j}$ whenever $t_{i j}=1$. If $t_{i j}=1$, there is thus a unique local inverse $\psi_{i j}: U_{j} \mapsto U_{i}$ off; the map $\psi_{i j}$ is $C^{r}$ and $d\left(\psi_{i j} x, \psi_{i j} y\right) \leqq \theta d(x, y)$. If $\xi \in \Omega$, the intersection $\bigcap_{n \geqq 0} f^{n} U_{\xi(n)}$
consists of the single point $\pi(\xi)$.

The proof of the existence of the $U_{i}$ is like the proof of Lemma 1.2, and the rest is easy. 
For the combinatorial considerations which follows, it is convenient to assume that $I$ is totally ordered. Let then $I(k)$ consist of the $k+1-$ tuples $\vec{i}=\left(i_{0}, i_{1}, \ldots, i_{k}\right)$ such that $i_{0}<i_{1}<\cdots<i_{k}$ and $X_{i_{0}} \cap \cdots \cap X_{i_{k}} \neq \varnothing$. Suppose that there is a permutation $\pi$ of $\vec{j}=\left(j_{0}, j_{1}, \ldots, j_{k}\right)$ such that

$$
f X_{i_{\ell}} \supset X_{\pi j_{\ell}} \text { for } \ell=0,1, \ldots, k \text {. }
$$

We then write

$$
t_{\vec{i} \vec{j}}=\left\{\begin{array}{lll}
+1 & \text { if } \pi \text { is even } \\
-1 & \text { if } \pi \text { is odd }
\end{array}\right.
$$

and $t_{\vec{i} \vec{j}}=0$ if there is no permutation $\pi$ as above.

Given an integer $m>0$, suppose that $\vec{i}_{0}, \vec{i}_{1}, \ldots, \vec{i}_{m-1}$ are given such that $t_{\vec{i}_{0} \vec{i}_{1}} \cdots t_{\vec{i}_{m-1} \vec{i}_{0}} \neq 0$, then there is a unique point $x\left(\vec{i}_{0}, \ldots, \vec{i}_{m-1}\right)$ in Fix $f^{m}$ such that

$$
f^{\ell} x\left(\vec{i}_{0}, \ldots, \vec{i}_{m-1}\right) \in U_{i_{\ell 0}} \cap \cdots \cap U_{i_{\ell k}}
$$

for $\ell=0, \ldots m-1$. [Fix $f^{m}$ denotes the set $\left\{x: f^{m} x=x\right\}$. The existence and uniquenss of $x\left(\vec{i}_{0}, \ldots, \vec{i}_{m-1}\right)$ follows from the existence of the local inverses $\psi_{i j}$, and the fact that they are contractions.] Note that, in fact, $x\left(\vec{i}_{0}, \ldots, \vec{i}_{m-1}\right) \in X_{i_{00}} \cap \ldots$ $\cap X_{i_{0 k}}$. We describe now the precise relation between fix $f^{m}$ and the points $x\left(\vec{i}_{0}, \ldots, \vec{i}_{m-1}\right)$.

2.4 Proposition (Counting periodic points).

$$
\sum_{k}(-1)^{k} \sum_{\left(\vec{i}_{0}, \ldots, \vec{i}_{m-1}\right): x\left(\vec{i}_{0}, \ldots, \vec{i}_{m-1}\right)=x} t_{\overrightarrow{\vec{b}}_{0} \vec{i}_{1}} \cdots t_{\vec{i}_{m-1} \vec{i}_{0}}= \begin{cases}1 & \text { if } x \in \text { Fix } f^{m} \\ 0 & \text { otherwise. }\end{cases}
$$

It suffices to discuss the case where $x \in \operatorname{Fix} f^{m}$. Let then $X_{i_{\ell} 1}, \ldots, X_{i_{\ell n \ell \ell},}$ be the $X_{i}$ which contain $f^{\ell} x$, for $\ell=0, \ldots, m-1$. Each $X_{i_{\ell+1) k}}$ is contained in a unique $f X_{i_{\ell k}}$ by the existence of local inverses and the Markov property (we take $l+1(\bmod m)$ so that $(m-1)+1=0)$. This implies that $n(\ell)$ is independent of $\ell$, and gives natural bijections

$$
\left(i_{01}, \ldots, i_{0 n}\right) \mapsto\left(i_{11}, \ldots, i_{1 n}\right) \mapsto \cdots \mapsto\left(i_{m-1,1}, \ldots, i_{m-1, n}\right) \mapsto\left(i_{01}, \ldots, i_{0 n}\right) .
$$

Let $\pi$ be the resulting permutation of $\left(i_{01}, \ldots, i_{0 n}\right)$. If $\pi$ is decomposed into disjoint cycles, the unions of cycles are the only sets $\vec{i}_{0}$ for which one has $x\left(\vec{i}_{0}, \ldots, \vec{i}_{m-1}\right)=x$. Furthermore, if $c$ is the number of cycles in $\vec{i}_{0}$, then $t_{\vec{i}_{0} \vec{i}_{1}} \cdots t_{\vec{i}_{m-1}} \vec{i}_{0}=(-1)^{c+k}$, and the proposition readily follows. Slightly different forms of the above counting argument have been given by Manning (11), and Bowen [4].

Markov partitions are a convenient tool, but they have the disadvantage of not being canonical. We shall be able to get rid of this arbitrariness in our main final results. A direct approach (not using Markov partitions) is sometimes useful, and will be developed elsewhere.

\section{Transfer Operators: Definition and Results}

Let $E$ be a smooth finite-dimensional real or complex vector bundle over $M$, and choose a smooth Riemann metric on $E$. If $\mathbf{r}$ is finite, the $C^{\mathbf{r}}$ sections of $E$ over $U$ 
which have bounded $C^{\mathrm{r}}$ norm form a Banach space $\mathscr{C}_{E}^{\mathrm{r}}(U)$. [Different Riemann metrics give equivalent norms if $U$ has compact closure in $M$, the choice of Riemann metric will thus be unimportant.] The $C^{\infty}$ sections form a Fréchet space

$$
\mathscr{C}_{E}^{\infty}(U)=\bigcap_{r \geqq 0} \mathscr{C}_{E}^{r}(U)
$$

For $C^{\omega}$ sections, it is convenient to consider those which extend to some given complex neighborhood $\tilde{U}$ of $U$, and to define a Banach space by using a norm on such extensions. (In this manner, $\mathscr{C}_{E}^{\omega}(U)$ is a countable union of Banach spaces.)

If $\varphi$ is a $C^{\mathrm{r}}$ bundle map over $f$, we define an operator $\mathscr{L}=\mathscr{L}_{\varphi}$ on $\mathscr{C}_{E}^{\mathrm{r}}(U)$ by

$$
(\mathscr{L} \Phi)(x)=\sum_{y: f y=x} \varphi(y) \Phi(y) .
$$

$\mathscr{L}$ is called transfer operator (it is related to the transfer matrices of equilibrium statistical mechanics).

For finite $\mathbf{r}, \mathscr{L}$ acts on a Banach space, and the theorems below estimate the spectral radius and essential spectral radius of $\mathscr{L}$ in terms of the pressure $P(\log |\varphi|)$. where $\log |\varphi|$ denotes the function $x \mapsto \log |\varphi(x)|$ on $U,|\varphi(x)|$ being the operator norm of $\varphi(x): E_{x} \mapsto E_{f x}$.

3.1 Theorem. The spectral radius of $\mathscr{L}$ is $\leqq \exp P(\log |\varphi|)$.

3.2 Theorem. The essential spectral radius of $\mathscr{L}$ is $\leqq \theta^{|r|} \exp P(\log |\varphi|)$.

This second theorem extends a result of Tangerman [27], who was the first to study transfer operators and zeta functions (see Sect. 6) in the $C^{\mathrm{r}}$ setting. For a slightly different approach see Pollicott [16] Appendix 1.

Theorems 3.1 and 3.2 are proved in Sect. 4.

3.3 Corollary. (i) Let $\mathscr{L}=\mathscr{L}^{\mathrm{r}}$ and $\mathscr{L}^{\mathrm{s}}$ denote the transfer operators on $\mathscr{C}_{E}^{\mathrm{r}}(U)$ and $\mathscr{C}_{E}^{\mathrm{s}}(U)$ defined by the same $f, \varphi$ of class $C^{\mathbf{r}}$, with $\mathbf{r}>\mathbf{s}$. In the domain $\left\{\lambda:|\lambda|>\theta^{|s|} \exp P(\log |\varphi|)\right\}$, the transfer operators $\mathscr{L}^{\mathbf{r}}$ and $\mathscr{L}^{\mathbf{s}}$ have the same eigenvalues with the same multiplicities and the same generalized eigenspaces (which consist of $C^{\mathrm{r}}$ sections). If $f, \varphi$ are $C^{\infty}$, it therefore makes sense to speak of the eigenvalues and eigenfunctions of $\mathscr{L}$ acting on $\mathscr{C}_{E}^{\infty}(U)$. In particular, if $f, \varphi$ are $C^{\omega}, \mathscr{L}$ acting on $C^{\infty}$ sections has $C^{\omega}$ eigenfunctions.

(ii) If $|\lambda|>\theta^{|r|} \exp P(\log |\varphi|)$, the elements of the generalized eigenspace of $\mathscr{L}^{*}$ corresponding to the eigenvalue $\lambda$ are distributions with support in $X$, of order sfor all

$$
s>\frac{P(\log |\varphi|)-\log |\lambda|}{|\log \theta|} .
$$

(iii) Define

$\mathscr{Z}^{r}=\left\{\Phi \in \mathscr{C}_{E}^{\mathrm{r}}(U): \Phi\right.$ and its derivatives up to order $r$ vanish identically on $\left.X\right\}$.

Then, the restriction of $\mathscr{L}$ to $\mathscr{Z}^{\text {r }}$ has spectral radius $\leqq \theta^{|r|} \exp P(\log |\varphi|)$. In particular, in $\left\{z:|z|>\theta^{|r|} \exp P(\log |\varphi|)\right\}$, the eigenvalues of $\mathscr{L}$ acting on the quotient $\mathscr{C}_{E}^{\mathrm{r}}(U) / \mathscr{Z}^{\mathrm{r}}$ (space of sections of the bundle of r-jets over $X$ defined by $\mathscr{C}^{\mathrm{r}}$ functions on $U$ ) are the same as the eigenvalues of $\mathscr{L}$ acting on $\mathscr{C}_{E}^{\mathrm{r}}(U)$ and have the same multiplicity.

This corollary is proved in Sect. 4. 
3.4 Remarks.

(i) One can, in Theorems 3.1 and 3.2, replace $P(\log |\varphi|)$ by

$$
P\left(\frac{1}{\ell} \log \left|\left(\varphi \circ f^{\ell-1}\right) \cdots(\varphi \circ f) \cdot \varphi\right|\right)
$$

for any integer $\ell \geqq 1$.

This follows readily from the proofs.

(ii) Let $r=\omega$, and replace $\varphi$ by a differential operator $(\varphi(y) \Phi(y)$ is thus an element of $E_{f y}$, linear combination of derivatives of $\Phi(y)$ with real-analytic coefficients). A form of Theorems 3.1 and 3.2 remains true: $\mathscr{L}$ is a compact operator on a suitable Banach space of complex extensions of sections of $E$.

In fact the methods of [18] extend to this case. One does not however expect Theorems 3.1 and 3.2 to extend in general to differential operators as above in the $C^{\infty}$ setting.

Let us now assume that $\mathbf{r} \geqq 1$, and let $T^{*} M$ be the cotangent bundle of $M$, and $\Lambda^{\ell}\left(T^{*} M\right)$ its $\ell$-th exterior power. The sections of $E \otimes \Lambda^{\ell}\left(T^{*} M\right)$ can be interpreted as $E$-valued $\ell$-forms, and there is a naturally defined bundle map $\varphi \otimes \Lambda^{\ell}\left(T^{*} f^{-1}\right)$ of $E \otimes \Lambda^{\ell}\left(T^{*} M\right)$ over $f$, which is of class $C^{r-1}$. We denote by $\mathscr{L}^{(\ell)}=\mathscr{L}_{\varphi}^{(\ell)}$ the corresponding transfer operator acting on $C^{\mathrm{r}-1} E$-valued $\ell$-forms; $\mathscr{L}^{(0)}$ is identified with $\mathscr{L}$.

3.5 Corollary. If $1 \leqq \ell \leqq \operatorname{dim} M$, the spectral radius of $\mathscr{L}^{(\ell)}$ is $\leqq \theta^{\ell} \exp P(\log |\varphi|)$ and the essential spectral radius of $\mathscr{L}^{(\ell)}$ is $\leqq \theta^{|r|+\ell-1} \exp P(\log |\varphi|)$.

This follows from Theorems 3.1 and 3.2 when $|\mathbf{r}|$ is replaced by $|\mathbf{r}|-1$ and $\exp P(\log |\varphi|)$ by $\theta^{\ell} \exp P(\log |\varphi|)$ [because $\left.\left|\varphi \otimes \Lambda^{\ell}\left(T^{*} f^{-1}\right) \leqq \theta^{\ell}\right| \varphi \mid\right]$.

If $E$ is the trivial one-dimensional bundle over $M$, we identify $\mathscr{C}_{E}^{r}(U)$ with the space $\mathscr{C}_{\mathbb{R}}^{\mathrm{r}}(U)$ or $\mathscr{C}_{\mathbb{C}}^{\mathrm{r}}(U)$ of $C^{\mathrm{r}}$ functions $U \mapsto \mathbb{R}$ or $\mathbb{C}$.

3.6 Theorem. Let $g \in \mathscr{C}_{\mathbb{R}}^{\mathrm{r}}(U)$, and let $\mathscr{L}_{g}$ act on $\mathscr{C}_{\mathbb{C}}^{\mathrm{r}}(U)$. $S \geqq 0$.

(i) If $g \geqq 0$, then $\exp P(\log g)$ is an eigenvalue of $\mathscr{L}_{g}$, and it has an eigenfunction

(ii) If $g$ is strictly positive, $\exp P(\log g)$ is a simple eigenvalue of $\mathscr{L}_{g}$ (respectively $\mathscr{L}_{g}^{*}$ ), with eigenfunction $S>0$ (respectively a measure $\sigma \geqq 0$ ) and all other eigenvalues have strictly smaller modules.

This theorem is proved in Sect. 4. We shall see later (Corollary 5.2) that if $g \geqq 0$ and $\exp P(\log g)$ is a simple eigenvalue of $\mathscr{L}_{g}$, the product $S \sigma$ of the corresponding eigenvectors of $\mathscr{L}_{g}$ and $\mathscr{L}_{g}^{*}$ is (up to normalization) the unique equilibrium state for $g$.

3.7 Theorem. Let $E$ be a real vector bundle, and suppose that there is a continuous family $\left(C_{x}\right)$ of closed strictly ${ }^{4}$ convex cones with nonempty interiors such that $C_{x} \subset E_{x}$, and $\varphi C_{x} \subset C_{f x}$.

${ }^{4}$ We say that the convex cone $C$ is strictly convex if $C \cap(-C)=\{0\}$ 
(i) The spectral radius of $\mathscr{L}$ is an eigenvalue of $\mathscr{L}$, and it has an eigenfunction $\Phi_{0}$ such that $\Phi_{0}(x) \in C_{x}$ for all $x$.

(ii) if $\varphi\left(C_{x} \backslash\left\{0_{x}\right\}\right) \subset \operatorname{int} C_{f x}$ for all $x$, the spectral radius $\lambda_{0}$ of $\mathscr{L}$ is a simple eigenvalue; its eigenfunction $\Phi_{0}$ satisfies $\Phi_{0}(x) \in \operatorname{int} C_{x}$ for all $x$. All other eigenvalues have modulus $<\lambda_{0}$.

This theorem is proved in Sect. 4.

\section{Transfer Operators: Proofs}

Before embarking in the proof of Theorems 3.1 and 3.2, we shall discuss the choice of the open set $U$, and introduce new operators $\mathscr{M}$ and $\mathscr{M}^{(m)}$ related to the transfer operator $\mathscr{L}$ defined by (3.1).

4.1 Remarks on the Choice of $U$. The operators obtained for sufficiently small neighborhoods $U$ of $X$ are in some sense equivalent: if $U^{\prime} \subset U$, then $\mathscr{L}^{m} \Phi$ is determined by $\Phi \mid U^{\prime}$ if $m$ is large enough. [Use the fact that $f$ is expanding.] More precisely, let $\mathscr{L}^{\prime}$ be the transfer operator corresponding to $U^{\prime}$, and $Q$ the operator restricting sections from $U$ to $U^{\prime}$. There is then a bounded operator $R: \mathscr{C}_{E}^{\mathrm{r}}\left(U^{\prime}\right) \mapsto$ $\mathscr{C}_{E}^{\mathrm{r}}(U)$ such that

$$
\begin{aligned}
Q \mathscr{L} & =\mathscr{L}^{\prime} Q, & \mathscr{L} R & =R \mathscr{L}^{\prime}, \\
Q R & =\mathscr{L}^{\prime m}, & R q & =\mathscr{L}^{m} .
\end{aligned}
$$

These relations ensure that the spectral theories of $\mathscr{L}$ and $\mathscr{L}^{\prime}$ are essentially the same.

4.2 The Operators $\mathscr{M}, \mathscr{M}^{(m)}$. It will be convenient to assume that $U=\bigcup_{i \in I} U_{i}$, where the $U_{i}$ satisfy Proposition 2.3. We may also assume that the vector bundle $E$ has trivial restriction $\cong U_{i} \times \mathbb{R}^{d}$ to each $U_{i}$.

We shall identify the $U_{i}$ with subsets of $\mathbb{R}^{\operatorname{dim} M}$. Taking the $U_{i}$ small enough we may (up to a small change of $\theta$ ) replace the Riemann metric by a Euclidean metric on $U_{i}$ if desired. We may also use the Euclidean metric on the fibres $\mathbb{R}^{d}$ of $U_{i} \times \mathbb{R}^{d}$.

Let $\sum_{i \in I} U_{i}$ be the disjoint union of the $U_{i}$. We may identify $\mathscr{C}_{E}^{\mathrm{r}}(U)$ with a subspace of

$$
\mathscr{C}_{E}^{\mathrm{r}}\left(\Sigma U_{i}\right)=\bigoplus_{i \in I} \mathscr{C}_{E}^{\mathrm{r}}\left(U_{i}\right)
$$

With this identification, $\mathscr{L}$ is the restriction to $\mathscr{C}_{E}^{\mathrm{r}}(U)$ of the operator $\mathscr{M}$ on $\mathscr{C}_{E}^{\mathrm{r}}\left(\Sigma U_{i}\right)$ defined as follows:

$$
(\mathscr{M} \Phi)_{j}(x)=\sum_{i: t_{i j}=1} \varphi\left(\psi_{i j} x\right) \Phi_{i}\left(\psi_{i j} x\right)
$$

where $\Phi_{i}=\Phi \mid U_{i}$, and $(\mathscr{M} \Phi)_{j}=\mathscr{M} \Phi \mid U_{j}$. In particular, the spectral radius of $\mathscr{L}$ is not larger than that of $\mathscr{M}$. Note that an eigenvalue $\lambda$ of $\mathscr{L}$ is also an eigenvalue of $\mathscr{M}$, and that the multiplicity with respect to $\mathscr{M}$ is at least the multiplicity with respect to $\mathscr{L}$. Note also that $\mathscr{C}_{E}^{\mathbf{r}}(U)$ is an invariant subspace for the resolvant 
$(1-z \mathscr{M})^{-1}$ when $|z|$ is small, and also (by analytic continuation) when $|z|$ is smaller than the inverse of the essential spectral radius and $z^{-1}$ is not an eigenvalue. Therefore, the essential spectral radius of $\mathscr{L}$ is not larger than that of $\mathscr{M}$.

We now combine the ideas described above to obtain an operator $\mathscr{M}^{(m)}$ which is in some sense equivalent to $\mathscr{L}^{m}$. We say that $\left(i_{0}, i_{1}, \ldots, i_{m}\right)$ is an allowed $m+1$-tuple if $t_{i_{0} i_{1}}=t_{i_{1} i_{2}}=\cdots=t_{l_{m-1} i_{m}}=1$. We let then

$$
\begin{gathered}
U\left(i_{0}, i_{1}, \ldots, i_{m}\right)=\psi_{i_{0} i_{1}} \circ \cdots \circ \psi_{i_{m-1} i_{m}} U_{i_{m}}, \\
\varphi_{\left(i_{0}, i_{1}, \ldots, i_{m}\right)}(x)=\varphi\left(\psi_{i_{m-1} i_{m}} x\right) \cdots \varphi\left(\psi_{i_{0} i_{1}} \cdots \psi_{i_{m-1} i_{m}} x\right) .
\end{gathered}
$$

The operator $\mathscr{M}^{(m)}: \mathscr{C}_{E}^{\mathrm{r}}\left(\Sigma U\left(i_{0}, \ldots, i_{m}\right)\right) \mapsto \mathscr{C}_{E}^{\mathrm{r}}\left(\Sigma U_{i}\right)$ is defined by

$$
\left(\mathscr{M}^{(m)} \Phi\right)_{i_{m}}(x)=\sum_{\left(i_{0}, \ldots, i_{m-1}\right)} \varphi_{\left(i_{0}, i_{1}, \ldots, i_{m}\right)}(x) \Phi\left(\psi_{i_{0} i_{1}} \cdots \psi_{i_{m-1} i_{m}} x\right),
$$

where the sum is over $m$-tuples such that $\left(i_{0}, \ldots, i_{m}\right)$ is allowed. (This formula reduces to (4.1) for $m=1$ ). If $Q^{(m)}$ is the operator which identifies $\mathscr{C}_{E}^{\mathrm{r}}\left(\sum_{i} U_{i}\right)$ with a subspace of $\mathscr{C}_{E}^{\mathrm{r}}\left(\Sigma U\left(i_{0}, \ldots, i_{m}\right)\right)$ (by restriction of sections) we have

$$
\mathscr{M}^{(m)} Q^{(m)}=\mathscr{M}^{m}
$$

4.3 The Operators $\mathscr{M}_{k}$. Given $k \geqq 0$, let

$$
\mathscr{C}_{E, k}^{\mathbf{r}}=\underset{\left(i_{0}, i_{1}, \ldots, i_{k}\right) \in I(k)}{\bigoplus_{E}} \mathscr{C}_{E}^{\mathbf{r}}\left(U_{i_{0}} \cap U_{i_{1}} \cap \cdots \cap U_{i_{k}}\right),
$$

where the sum is over all $k+1$ - tuples $\vec{i}=\left(i_{0}, i_{1}, \ldots, i_{k}\right)$ with $i_{0}<i_{1}<\cdots<i_{k}$ such that $U_{i_{0}} \cap U_{i_{1}} \cap \cdots \cap U_{i_{k}} \neq \varnothing$. We define an operator $\mathscr{M}_{k}$ on $\mathscr{C}_{E, k}^{\mathrm{r}}$ by

$$
\left(\mathscr{M}_{k} \Phi\right)_{\vec{j}}(x)=\sum_{\vec{i}: t_{i} \vec{j}= \pm 1} t_{\vec{i} \vec{j}} \varphi\left(\psi_{\vec{i} \vec{j}} x\right) \Phi_{\vec{i}}\left(\psi_{\vec{i} \vec{j}} x\right),
$$

where $t_{\bar{\imath} \vec{j}}$ has been defined in Sect. 2 and $\psi_{\vec{i} \vec{j}}$ is the local inverse of $f$ which maps $U_{j_{0}} \cap \cdots \cap U_{j_{k}}$ into $U_{i_{0}} \cap \cdots \cap U_{i_{k}}$. Clearly, $\mathscr{M}_{0}$ can be identified with $\mathscr{M}$. The reader will easily figure how to define $\mathscr{M}_{k}^{(m)}$ by analogy with the operator $\mathscr{M}^{(m)}$ of Eq. (4.4).

4.4 Remark. The operators $\mathscr{M}_{k}$ are of the same type as $\mathscr{M}=\mathscr{M}_{0}$, with the replacement of $\left(X_{i}\right)$ by $\left(X_{i_{0}} \cap \cdots \cap X_{i_{k}}\right)$ and of $\varphi \circ \psi_{i j}$ by $t_{\vec{i} j} \varphi \circ \psi_{\vec{i} j}$. Note that $\left|t_{\vec{i} \vec{j}} \cdot \varphi \circ \psi_{\vec{i} \vec{j}}\right|=\left|\varphi \circ \psi_{\vec{i} \vec{j}}\right|$, and that $\bigcup_{\left(i_{0}, \ldots, i_{k}\right) \in I(k)} X_{i_{0}} \cap \cdots \cap X_{i_{k}}$ is a subset of $X$. It then follows from symbolic dynamics (see next lemma) that in going from $\mathscr{M}$ to $\mathscr{M}_{k}$ one has to replace $\exp P(\log |\varphi|)$ by a quantity $\leqq \exp P(\log |\varphi|)$.

4.5 Lemma. If $g: U \mapsto \mathbb{R}$ is continuous $\geqq 0$,

$$
\lim _{M \rightarrow \infty} \frac{1}{m} \log \sup _{i_{m}} \sup _{x \in U_{i m}} \sum_{i_{0}, i_{1}, \ldots, i_{m-1}} g_{\left(i_{0}, \ldots, i_{m}\right)}(x)=P(\log g) .
$$

(The $\left.g_{\left(i_{0}, \ldots, i_{m}\right.}\right)$ are defined by (4.3).)

If $g$ does not vanish, write $g=\exp A$ with continuous $A: U \mapsto \mathbb{R}$. we may replace $x \in U_{i_{m}}$ by $x \in X_{i_{m}}$, the lemma is then an easy consequence of symbolic dynamics. 
[See for instance [20], Chap. 7, note that $A \circ \pi$ can be approximated by a function of a finite sequence of symbols.]

In the general case, let $\left(A_{\ell}\right)$ be a decreasing sequence tending to $\log g$. The expression

$$
\frac{1}{m} \log \sup _{i_{m}} \sup _{x \in U_{i m}} \sum_{i_{0}, \ldots, i_{m-1}} g_{\left(i_{0}, \ldots, i_{m}\right)}^{\ell}(x),
$$

where $g_{\left(i_{0}, \ldots, i_{m}\right)}^{\ell}$ corresponds to the replacement of $g$ by exp $A_{\ell}$, is a subadditive function of $m$, and a decreasing function of $\ell$. We may thus interchange the limits $m \rightarrow \infty, \ell \rightarrow \infty$. To conclude, notice that

$$
\lim _{\ell \rightarrow \infty} P\left(A_{\ell}\right)=\lim _{\ell \rightarrow \infty} \max _{\rho \in \mathbf{I}}\left(h(\rho)+\rho\left(A_{\ell}\right)\right)=\max _{\rho \in \mathbf{I}}(h(\rho)+\rho(\log g))=P(\log g) .
$$

(Confer the proof of Lemma 1.4.)

4.6 Proof of Theorem 3.1. Since the spectral radius of $\mathscr{L}$ is bounded above by that of $\mathscr{M}$, it suffices to study $\lim \left(\left\|\mathscr{M}^{m}\right\|\right)^{1 / m}$. In view of (4.5) and of the bound $\left\|Q^{(m)}\right\| \leqq 1$, we are reduced to proving that

$$
\lim _{m \rightarrow \infty}\left(\left\|\mathscr{M}^{(m)}\right\|\right)^{1 / m} \leqq \exp P(\log |\varphi|) .
$$

Let us first estimate the $C^{0}$ norm of $\mathscr{M}^{(m)} \Phi$. For any $\varepsilon>0$ we can write, in view of the above lemma,

$$
\begin{aligned}
\left\|\mathscr{M}^{(m)} \Phi\right\|_{0} & \leqq \sup _{i_{m}} \sup _{x \in U_{i_{m}}} \sum_{i_{0}, \ldots, i_{m-1}}\left|\varphi_{\left(i_{0}, \ldots, i_{m}\right)}(x)\right| \cdot\|\Phi\| \\
& \leqq\|\Phi\| \cdot \text { const } \exp m(P(\log |\varphi|)+\varepsilon) .
\end{aligned}
$$

We now have to estimate the derivatives of $\mathscr{M}^{(m)} \Phi$; differentiating (4.4) $\ell$ times we obtain a variety of terms, but the number of terms is polynomial in $m$, and each term has an estimate similar to (4.6), so that

$$
\left\|\mathscr{M}^{(m)} \Phi\right\|_{\ell} \leqq\|\Phi\| \cdot \mathscr{P}_{\ell}(m) \exp m(P(\log |\varphi|)+\varepsilon),
$$

where $\mathscr{P}_{\ell}$ is a polynomial. The estimate of the Hölder norm of the $r^{\text {th }}$ derivative is similar, therefore

$$
\left\|\mathscr{M}^{(m)}\right\| \leqq \mathscr{P}(m) \exp m(P(\log |\varphi|+\varepsilon),
$$

and the theorem follows.

4.7 Proof of Theorem 3.2. The essential spectral radius of $\mathscr{L}$ is bounded above by that of $\mathscr{M}$, which we shall estimate by use of Nussbaum's formula [12]. This formula gives for the essential spectrum of a bounded linear operator $\mathscr{M}$ on a Banach space the upper bound

$$
\liminf _{m \rightarrow \infty}\left\|\mathscr{M}^{m}-K_{m}\right\|^{1 / m}
$$

where $\left(K_{m}\right)$ is a sequence of compact operators. In the present case, it suffices to 
show that

$$
\liminf _{m \rightarrow \infty}\left\|\mathscr{M}^{(m)}-K_{(m)}\right\|^{1 / m} \leqq \theta^{|\mathbf{r}|} \exp P(\log |\varphi|),
$$

where $\mathscr{M}^{(m)}$ is defined by (4.4) and $K_{(m)}$ has finite rank for each $m$.

We have identified the $U_{i}$ with subsets of Euclidean space, and we may identify sections of $E$ over $U_{i}$ with functions $U_{i} \mapsto \mathbb{R}^{d}$ or $\mathbb{C}^{d}$, where $d$ is the dimension of the fiber of $E$; the $\varphi_{\left(i_{0}, \ldots, i_{m}\right)}$ become thus matrix-valued functions. In this setup we shall use Taylor expansions to define the operators $K_{(m)}$.

Choose $\bar{x}\left(i_{0}, \ldots, i_{m}\right) \in U\left(i_{0}, \ldots, i_{m}\right)$ for each allowed $m+1$-tuple $\left(i_{0}, \ldots, i_{m}\right)$. We define $K_{(m)}=\mathscr{M}^{(m)} \mathscr{J}_{r}$, where $\mathscr{J}_{r} \Phi$ is the Taylor expansion of $\Phi$ to order $r$ at $\bar{x}\left(i_{0}, \ldots, i_{m}\right)$ in the set $U\left(i_{0}, \ldots, i_{m}\right)$ defined by (4.2) and considered as an open set in Euclidean space. We let $\mathscr{R}$ be the remainder of the Taylor expansion, so that

$$
\left(\left(\mathscr{M}^{(m)}-K_{(m)}\right) \Phi\right)_{i_{m}}(x)=\sum_{i_{0}, \ldots, i_{m-1}} \varphi_{\left(i_{0}, \ldots, i_{m}\right)}(x) \mathscr{R}_{\left(i_{0}, \ldots, i_{m}\right)}\left(\psi_{i_{0} i_{1}} \cdots \psi_{i_{m-1} i_{m}} x\right) .
$$

Integrating

$$
\left|\partial^{r} \mathscr{R}(x)\right| \leqq\|\Phi\| \cdot\left(x-\bar{x}\left(\left(i_{0}, \ldots, i_{m}\right)\right)^{\alpha}\right.
$$

we find

$$
|\mathscr{R}(x)| \leqq \text { const } \cdot\|\Phi\|\left(x-\bar{x}\left(\left(i_{0}, \ldots, i_{m}\right)\right)^{|\mathbf{r}|}\right.
$$

for $x \in U\left(i_{0}, \ldots, i_{m}\right)$, hence

$$
\|\mathscr{R}\|_{0} \leqq \text { const } \cdot\|\Phi\| \theta^{m|\mathbf{r}|},
$$

and therefore, using Lemma 4.5,

$$
\begin{aligned}
\left\|\left(\mathscr{M}^{(m)}-K_{(m)}\right) \Phi\right\|_{0} & \leqq \text { const } \cdot\|\Phi\| \theta^{m|\mathbf{r}|} \sup _{i_{m}} \sup _{x \in U_{i_{m}}} \sum_{i_{0}, \ldots, i_{m-1}}\left|\varphi_{\left(i_{0}, \ldots, i_{m}\right)}(x)\right| \\
& \leqq\|\Phi\| \text { const } \cdot \theta^{m|\mathbf{r}|} \exp (m P(\log |\varphi|+\varepsilon)) .
\end{aligned}
$$

Estimating the derivatives of $\left(\mathscr{M}^{(m)}-K_{(m)}\right) \Phi$, and the Hölder norm of the $r^{\text {th }}$ derivative yield a similar result (up to polynomials in $m$, see the proof of Theorem 3.1). Therefore

$$
\left\|\mathscr{M}^{(m)}-K_{(m)}\right\| \leqq \mathscr{P}(m) \theta^{m|\mathbf{r}|} \exp (m P(\log |\varphi|+\varepsilon)),
$$

and the theorem follows.

4.8 Proof of Corollary 3.3. Let $\mathbf{s}<\mathbf{r}$ finite, so that $\mathscr{C}_{R}^{\mathbf{r}}(U)$ and $\mathscr{C}_{E}^{\mathbf{s}}(U)$ are Banach spaces. We have $\mathscr{C}_{E}^{\mathrm{r}}(U) \subset \mathscr{C}_{E}^{\mathrm{s}}(U)$, and the generalized eigenspace $\mathscr{E}_{\lambda}^{\mathbf{r}}(U)$ of $\mathscr{L}^{\mathbf{r}}$ corresponding to the eigenvalue $\lambda$ is a subspace of $\mathscr{E}_{\lambda}^{\text {s }}$. Correspondingly, the canonical map $\mathscr{C}_{E}^{\mathbf{s}}(U)^{*} \mapsto \mathscr{C}_{E}^{\mathrm{r}}(U)^{*}$ sends $\mathscr{E}_{\lambda}^{\mathscr{s}^{*}}$ onto $\mathscr{E}_{\lambda}^{\mathrm{r} *}$ (because of duality with the injective map $\mathscr{E}_{\lambda}^{\mathrm{r}} \mapsto \mathscr{E}_{\lambda}^{\mathrm{s}}$ ). To prove Part (a) of the corollary, it suffices to show that if $\sigma \in \mathscr{E}_{\lambda}^{\mathbf{s} *}$ and the image $\sigma^{\mathbf{r}}$ of $\sigma$ in $\mathscr{E}_{\lambda}^{\mathbf{r} *}$ vanishes, then $\sigma=0$. We choose $\mathbf{t}$ with $|\mathbf{t}|<|\mathbf{s}|$, such that $|\lambda|>\theta^{|\mathbf{t}|} \exp P(\log |\varphi|)$, and we extend $\sigma$ to an element $\sigma^{\mathbf{t}}$ of $\mathscr{E}_{\lambda}^{\mathbf{t} *}$. Using the fact that $\sigma$ is a (generalized) eigenfunction, and the existence of local inverses for the expanding map $f$, we see that $\sigma(\Phi)$ depends only on the restriction of $\Phi$ to an arbitrarily small neighborhood of $X$. If $\sigma \neq 0$, there is thus $\Phi$ of class $C^{\text {s }}$ with compact support in $U$ such that 
$\sigma(\Phi) \neq 0$. But, by regularization, we may write $\Phi=\lim \Phi_{i}$, where the $\Phi_{i}$ are of class $C^{\mathrm{r}}$ and converge to $\Phi$ in the $C^{\mathbf{t}}$ topology. Then,

$$
\sigma(\Phi)=\sigma^{\mathrm{t}}(\Phi)=\lim \sigma^{\mathrm{t}}\left(\Phi_{i}\right)=\lim \sigma^{\mathrm{r}}\left(\Phi_{i}\right)=0 .
$$

This proves Part (i) of the corollary for finite $\mathbf{r}$, and the assertion still makes sense for $\mathbf{r}=\infty$ since

$$
\mathscr{C}_{E}^{\infty}=\bigcap_{r \text { finite }} \mathscr{C}_{E}^{r}(U)
$$

If $\mathbf{r}=\omega$, we have again injectivity of $\mathscr{E}_{\lambda}^{\mathbf{r}} \rightarrow \mathscr{E}_{\lambda}^{\mathbf{s}}$, and we may use the same argument as for finite $\mathbf{r}$.

Taking $\sigma$ as above, we see that it defines a continuous linear functional on $C^{\infty}$ functions in $U$, i.e., a distribution in the sense of Schwartz. We have seen that this distribution has compact support contained in $X$; it has therefore finite rank $k$, i.e., it extends uniquely from the $C^{\infty}$ functions to the $C^{k}$ functions. By part (i) of the corollary it extends then uniquely to $C^{s}$ functions, where $s$ satisfies (3.2). This proves Part (ii) of the corollary.

Let $\mathscr{Z}^{r}$ be the subspace of $\mathscr{C}_{E}^{\mathrm{r}}(U)$ consisting of those $\Phi$ 's which vanish on $X$ together with their derivatives up to order $r$. We have then $\mathscr{L}^{2} \subset \mathscr{Z}^{r}$. Furthermore, the proof of Theorem 3.1 shows that the spectral radius of the restriction of $\mathscr{L}$ to $\mathscr{Z}^{r}$ is $\leqq \theta^{|\mathbf{r}|} \exp P(\log |\varphi|)$. From this, Part (iii) of the corollary follows.

4.9 Proof of Theorem 3.6. To study $\mathscr{L}_{g}$ when $g \geqq 0$, we appeal to symbolic dynamics (see Proposition 2.2, and $[3,20]$ ). Define a metric on $\Omega$ by

$$
\left.d\left(\left(\xi_{n}\right),(\eta)_{n}\right)\right)=\left\{\inf \theta^{k}: \xi_{n}=\eta_{n} \text { for } n<k\right\},
$$

then $\pi: \Omega \rightarrow X$ is Lipschitz. We write $\beta=\alpha$ if $\mathbf{r}=(0, \alpha), \beta=1$ if $|\mathbf{r}| \geqq 1$, and introduce the transfer operator $L$ on $\mathscr{C}_{\mathbb{C}}^{\beta}(\Omega)$ associated with the function $g \circ \pi$. Applying Theorem 3.2 to $L$ gives

$$
\text { ess } \cdot \text { spectral radius } L \leqq R(\mathbf{r})
$$

where we have written

$$
R(\mathbf{r})=\theta^{\beta} \cdot e^{P}=\left\{\begin{array}{lll}
\theta^{\alpha} e^{P} & \text { if } & \mathbf{r}=(0, \alpha) \\
\theta e^{P} & \text { if } & |\mathbf{r}| \geqq 1
\end{array}\right.
$$

and $P=P(\log g)$.

We have

$$
L(\Phi \circ \pi)=(\mathscr{L} \Phi) \circ \pi .
$$

Note that $\Phi \circ \pi=0$ if and only if $\Phi \mid X=0$. Furthermore, Corollary 3.3 (iii) shows that $\mathscr{L}$ restricted to $\{\Phi: \Phi \mid X=0\}$ has spectral radius $\leqq R(\mathbf{r})$. Therefore, (4.9) shows that the eigenvalues of $\mathscr{L}$ with modulus $>R(\mathbf{r})$ are also eigenvalues of $L$ (and the multiplicities for $\mathscr{L}$ are $\leqq$ the multiplicities for $L$ ).

The operator $L$ has been much studied in the case where $g$ is strictly positive (see [20]), and it is known that $\exp P$ is then a simple eigenvalue of $L$, corresponding to a strictly positive eigenfunction, and all other eigenvalues have strictly smaller modulus. It follows that 


$$
S=\lim _{m \rightarrow \infty} e^{-m P} \mathscr{L}^{m_{1}}>0 .
$$

$S$ is an eigenvalue of $\mathscr{L}$ to the eigenvalue $\exp P$, and the latter is simple, all the other eigenvalues of $\mathscr{L}$ having strictly smaller modulus. The eigenfunction $\omega$ of $L^{*}$ to the eigenvalue $\exp P$ is a positive measure, the same is therefore true of the eigenfunction $\sigma=\pi \omega$. This proves Part (ii) of the theorem. ${ }^{5}$

If $g \geqq 0$, Lemma 4.5 shows that

$$
\lim _{m \rightarrow \infty}\left(\left\|\mathscr{L}^{m_{1}}\right\|_{0}\right)^{1 / m}=e^{P} .
$$

This (together with Theorem 3.1) implies that the spectral radius of $\mathscr{L}$ is $e^{P}$. Let $\Phi \geqq 0$ and write

$$
\Phi=\Psi+\sum_{j} \Psi_{j}
$$

where, for each $j, \lambda_{j}$ is an eigenvalue of $\mathscr{L}$ with $\left|\lambda_{j}\right|=e^{P}$, and $\Psi_{j}$ is in the corresponding generalized eigenspace; $\Psi$ is such that

$$
\lim _{m \rightarrow \infty} \frac{\left\|\mathscr{L}^{m} \Psi\right\|}{\tilde{\lambda}^{m}}=0
$$

for some $\tilde{\lambda} \in\left(0, e^{P}\right)$. We may assume that not all $\Psi_{j}$ vanish (this is the case for instance if $\Phi=1)$. Using Jordan normal forms one sees that there is an integer $k \geqq 0$ such that

and

$$
\lim _{m \rightarrow \infty} \frac{1}{\lambda_{j}^{m} \cdot m^{k}} \mathscr{L}^{m} \Psi_{j}=\Phi_{j}
$$

$$
\mathscr{L} \Phi_{j}=\lambda_{j} \Phi_{j}
$$

for all $j$, and $\Phi_{j} \neq 0$ for some $j$. Using (4.10), (4.11) we find

$$
\sum_{j}\left(\frac{\lambda_{j}}{e^{P}}\right)^{m} \Phi_{j} \geqq-\varepsilon(m),
$$

where $\varepsilon(m) \rightarrow 0$ when $m \rightarrow \infty$. Remember that the sum over $j$ is finite, and that $\left|\lambda_{j} / e^{P}\right|=1$. Writing $\lambda_{0}=e^{P}$, we see from (4.12) that $\Phi_{0} \geqq 0$, and that $\Phi_{0}$ cannot vanish identically. This proves Part (i) of the theorem.

4.10 Proof of Theorem 3.7. The proof of (i) is analogous to that of Theorem 3.6 (i). In fact, according to Remark 3.4 (i), the spectral radius of $\mathscr{L}$ is

$$
\leqq \lambda_{0}=\lim _{\ell \rightarrow \infty} \exp P\left(\frac{1}{\ell} \log \left|\left(\varphi \circ f^{\ell-1}\right) \cdots(\varphi \circ f) \varphi\right|\right) \text {. }
$$

If $\Phi$ is chosen such that $\Phi(x) \in C_{x} \backslash\left\{0_{x}\right\}$ for all $x$, one sees (using the strict convexity of $C_{x}$ and $\varphi C_{x} \subset C_{f x}$ ) that $1 / m\left\|\mathscr{L}^{m} \Phi\right\|_{0} \rightarrow \log \lambda_{0}$. The same argument as in the

\footnotetext{
5 It follows from symbolic dynamics that the product $S \sigma$ is (up to normalization) the unique equilibrium state for $g$. We shall prove this results more generally in Corollary 5.2.
} 
proof of Theorem 3.6 (i) then shows that there is an eigenfunction $\Phi_{0}$ to the eigenvalue $\lambda_{0}$, such that $\Phi_{0}(x) \in C_{x}$ for all $x$. This proves (i).

To prove (ii) note first that the eigenfunction $\Phi_{0}$ does not vanish anywhere on $X$ because if $\Phi_{0}(x)=0$, then $\Phi$ vanishes on $\left\{y: f^{m} y=x\right.$ for some $\left.m\right\}$, which is dense in $X$ [use symbolic dynamics]. Therefore, we may assume that $\Phi_{0}$ does not vanish in $U$. For the rest of the proof we may forget about differentiability and restrict attention to Hölder continuous functions on $X$.

Let $C_{x}^{*}$ be the cone dual to $C_{x}$. Then, $\varphi(x)^{*} C_{f x}^{*} \subset C_{x}^{*}$. There is therefore a family $\left(L_{x}^{*}\right)$ of half-lines with $L_{x}^{*} \subset C_{x}^{*}$ and $\varphi(x)^{*} L_{f x}^{*}=L_{x}^{*}$. A standard hyperbolicity argument shows that $x \mapsto L_{x}^{*}$ is Hölder continuous on $X$ (see for instance [23] Part 2, Problem 4). Let $H_{x}$ be the hyperplane in $E_{x}$ defined by $L_{x}$. The sections $\Psi$ of the subbundle $H=\left(H_{x}\right)$ form an invariant subspace $\mathscr{H}$ for the operator $\mathscr{L}$. Furthermore, our assumption about $\varphi C_{x}$ implies that

$$
\frac{\left|\mathscr{L}^{m} \Psi(x)\right|}{\left|\mathscr{L}^{m} \Phi(x)\right|} \rightarrow 0 \quad \text { exponentially }
$$

(uniformly in $x$ ) when $m \rightarrow \infty$, hence

$$
\lambda_{0}^{-m}\left\|\mathscr{L}^{m} \mid \mathscr{H}\right\|_{0} \rightarrow 0 \text { exponentially. }
$$

We may apply Theorem 3.6 (ii) to the bundle map $\varphi^{+}$induced by $\varphi$ on the trivial one-dimensional quotient bundle $E^{+}=E / H$. The corresponding transfer operator $\mathscr{L}^{+}$thus has a simple positive eigenvalue of highest modulus $\leqq \lambda_{0}$. The generalized eigenspace of $\mathscr{L}$ to an eigenvalue $\lambda_{j}$ with $\left|\lambda_{j}\right|=\lambda_{0}$ maps injectively (because of (4.13)) into the generalized eigenspace of $\mathscr{L}^{+}$to the eigenvalue $\lambda_{j}$. Therefore $\lambda_{0}$ must be a simple eigenvalue of $\mathscr{L}$, and all other eigenvalues must have strictly smaller modulus. This proves (ii).

\section{Gibbs Distributions}

In [22] certain distributions in the sense of L. Schwartz have been introduced, called Gibbs distributions, which extend the notion of equilibrium state, and are useful (among other things) in the study of correlation functions. In the present setting, the Gibbs distributions take the simple form $\Phi \cdot \Psi$, where $\Phi$ and $\Psi$ are (generalized) eigenfunctions of $\mathscr{L}_{\varphi}$ and $\mathscr{L}_{\varphi}^{*}$ respectively, and $\Phi \cdot \Psi$ is the linear form on $\mathscr{C}_{\mathbb{C}}^{\mathrm{r}}(U)$ defined by $(\Phi \cdot \Psi)(A)=\Psi(A \Phi)$. [More generally one could take $A(x) \in$ End $E_{x}$, with $x \mapsto A(x)$ of class $C^{\mathrm{r}}$ on $U$ ]. Note that in [22] the distributions considered were in the dual of a space of Hölder continuous functions; here however we may have distributions of arbitrarily high order. Note also that Tangerman's idea (see [27]) of studying transfer matrices on spaces of differentiable functions brings rewards even when one can use spaces of analytic functions (see [18]); this is because Gibbs distributions appear as distributions of finite order rather than linear functionals on analytic functions.

5.1 Proposition. Let $A \in \mathscr{C}_{\mathbb{C}}^{\mathrm{r}}(U)$. [More generally one can take $A(x) \in$ End $E_{x}$, with $x \mapsto A(x)$ of class $C^{\mathrm{r}}$ on $\left.U\right]$. If $\lambda_{0}$ is a simple eigenvalue of $\mathscr{L}_{\varphi}$, and $\left|\lambda_{0}\right|>\exp P(\log |\varphi|)$. $\theta^{|\mathbf{r}|}$, there is an eigenvalue $\lambda(A)$ of $\mathscr{L}_{\varphi \exp A}$, depending holomorphically on $A$ in $a$ 
neighborhood of 0 in $\mathscr{C}_{\mathbb{C}}^{\mathrm{r}}(U)$, and such that $\lambda(0)=\lambda_{0}$. Furthermore the logarithmic derivative of $\lambda$ at 0 is given by $\left(\lambda^{-1} D_{0} \lambda\right) A=\Psi(A \Phi)$, where $\Phi, \Psi$ are the eigenvectors of $\mathscr{L}_{\varphi}, \mathscr{L}_{\varphi}^{*}$ for the eigenvalue $\lambda$, normalized so that $\Psi(\Phi)=1$. (We may thus write $\left.\lambda^{-1} D_{0} \lambda=\Phi \cdot \Psi\right)$.

It is easily seen that $A \mapsto \mathscr{L}_{\varphi \exp A}$ is holomorphic. Therefore one can choose the eigenvalue $\lambda(A)$ to depend holomorphically on $A$ in a neighborhood of 0 . Let also $\Phi(A), \Psi(A)$ be eigenvectors of $\mathscr{L}_{\lambda \exp A}, \mathscr{L}_{\lambda \exp A}^{*}$ to the eigenvalue $\lambda(A)$. We may again assume that $A \mapsto \Phi(A), \Psi(A)$ are holomorphic, and that $\Psi(A)(\Phi(A))=1$ identically. Writing $\partial^{A} \cdots=\left(D_{0} \cdots\right) A$, we have for the derivative of $\lambda$

$$
\begin{aligned}
\partial^{A} \lambda & =\partial^{A}(\Psi \mathscr{L} \Phi)=\left(\partial^{A} \Psi\right) \lambda \Phi+\lambda \Psi\left(\partial^{A} \Phi\right)+\Psi\left(\left(\partial^{A} \mathscr{L}_{\varphi \exp A}\right) \Phi\right) \\
& =\lambda \partial^{A}(\Psi \Phi)+\Psi(\mathscr{L}(A \Phi))=\left(\mathscr{L}^{*} \Psi\right)(A \Phi)=\lambda \Psi(A \Phi) .
\end{aligned}
$$

5.2 Corollary. Let $g \geqq 0$ and $\exp P(\log g)$ be a simple eigenvalue of $\mathscr{L}_{g}$ (this is the case in particular if $g$ does not vanish). Then $D_{\log g} P=S \cdot \sigma$, where $S, \sigma$ are the eigenvectors of $\mathscr{L}_{g}, \mathscr{L}_{g}^{*}$ for the eigenvalue $\exp P(\log g)$, normalized so that $\sigma(S)=1$. Furthermore, $\rho=S \cdot \sigma$ is the unique equilibrium state for $\log g$.

Note that if $\rho$ is an equilibrium state for $\log g$ we have, by Lemma 1.4,

$$
P(\log g+A) \geqq h(\rho)+\rho(\log g)+\rho(A)=P(\log g)+\rho(A) .
$$

But, because of the differentiability of $A \mapsto P(\log g+A)$, there can be only one $\rho$ with $\rho(A) \leqq P(\log g+A)-P(\log g)$ for all $A \in \mathscr{C}_{\mathbb{R}}^{\mathrm{r}}(U)$, and it is given by the derivative $D_{\log g} P=S \cdot \sigma$.

5.3 Proposition. Let $g \geqq 0$, write $P=P(\log g)$, and assume that $e^{P}$ is a simple eigenvalue of $\mathscr{L}_{g}, \mathscr{L}_{g}^{*}$ with eigenvectors $S, \sigma$ such that $\sigma(S)=1$. If $A, B \in \mathscr{C}_{\mathbb{C}}^{\text {r }}(U)$, the series 6

$$
S_{A B}(z)=\sum_{m=0}^{\infty} z^{m}\left[\rho\left(A \cdot\left(B \circ f^{m}\right)\right)-\rho(A) \rho(B)\right]
$$

converges for $|z|<\mid$, and extends to a meromorphic function in $\left\{z:|z|<\theta^{-|\mathbf{r}|}\right\}$, with poles at $\lambda^{-1} e^{\boldsymbol{P}}$, where $\lambda$ runs over the eigenvalues $\neq e^{\boldsymbol{P}}$ of $\mathscr{L}_{g}$. If $\lambda$ is a simple eigenvalue, and if $\Phi, \Psi$ are the corresponding eigenvectors of $\mathscr{L}_{g}, \mathscr{L}_{g}^{*}$ such that $\Psi(\Phi)=1$, then $\lambda e^{-P}$ is a simple pole of $S_{A B}$, with residue $\Psi(S A) \cdot \sigma(\Phi B)$.

This is based on a simple calculation which we present in a more general form than needed for the present problem. Let $\sigma$ be an eigenfunction of $\mathscr{L}^{*}$ to the eigenvalue $\lambda_{0}$. Then

$$
\sigma\left(A^{\prime} \cdot\left(B \circ f^{m}\right)\right)=\lambda_{0}^{-m} \mathscr{L}^{* m} \sigma\left(A^{\prime} \cdot\left(B \circ f^{m}\right)\right)=\lambda_{0}^{-m} \sigma\left(\left(\mathscr{L}^{m} A^{\prime}\right) \cdot B\right) .
$$

For the simplicity of formulae, let us assume that the eigenvalues $\lambda$ of $\mathscr{L}$ with modulus $>\theta^{|\mathbf{r}|} e^{P+\varepsilon}$ are simple, and denote by $\mathscr{P}$ the projection corresponding to the part of the spectrum of $\mathscr{L}$ in $\left\{\lambda:|\lambda| \leqq \theta^{|\mathbf{r}|} e^{P+\varepsilon}\right\}$. Then,

\footnotetext{
6 This series is essentially the Fourier transform of the correlation function $m \mapsto \rho\left(A .\left(B \circ f^{m}\right)\right)-\rho(A) \rho(B)$ associated with the equilibrium state
} 


$$
\begin{aligned}
\sum_{m=0}^{\infty} z^{m} \sigma\left(A^{\prime} \cdot\left(B \circ f^{m}\right)\right) & =\sigma\left(\left(\left(1-z \lambda_{0}^{-1} \mathscr{L}\right)^{-1} A^{\prime}\right) \cdot B\right) \\
& =\sum_{\lambda} \frac{\Psi\left(A^{\prime}\right) \sigma(\Phi B)}{1-z \lambda_{0}^{-1} \lambda}+\sigma_{0}\left(\left(\left(1-z \lambda_{0}^{-1} \mathscr{L}\right)^{-1} \mathscr{P} A^{\prime}\right) \cdot B\right) .
\end{aligned}
$$

If we take $\lambda_{0}=e^{P}$ and $A^{\prime}=S(A-\rho(A))$, the term with $\lambda=e^{P}$ disappears in the right-hand side, and the proposition follows.

Note that various generalizations are possible, where $A \in \mathscr{C}_{F}^{\mathrm{r}}(U), B \in \mathscr{C}_{F^{*}}^{\mathrm{r}}(U)$ and $F, F^{*}$ are dual bundles, or where $\lambda_{0} \neq e^{P}$, or where $g$ is complex or replaced by $\varphi \in \mathscr{C}_{E}^{\mathrm{r}}(U)$, etc.

\subsection{Remarks on $S_{A B}$.}

(i) If $\mathrm{g}>0, S_{A B}(z)$ converges for $|z| \leqq 1$.

(ii) In general, $S_{A B}$ may have multiple poles.

(iii) If $\mathbf{r}=\infty, S_{A B}$ is meromorphic in $\mathbb{C}$.

(iv) We have

$$
\left.\frac{\partial^{2}}{\partial s_{1} \partial s_{2}} P\left(\log g+s_{1} A+s_{2} B\right)\right|_{s_{1}=s_{2}=0}=S_{A B}(1)+S_{B A}(1)-\rho(A B) .
$$

The proof of (iv) is a simple calculation. [Let $\Phi, \Psi$ be eigenvectors of $\mathscr{L}_{\log g+s A} \mathscr{L}_{\log g+s A}^{*}$ such that $\Psi(\Phi)=1$. Differentiate $s \mapsto \Psi(B \Phi)$, where the derivatives of $\Phi, \Psi$ are obtained by differentiation of the equations $\mathscr{L} \Phi=\lambda \Phi, \mathscr{L}^{*} \Psi=\lambda \Psi$.]

\subsection{Remarks on Gibbs States.}

(i) In the generalization where $\varphi$ is replaced by a differential operator (see Remark 3.4 (ii)), one cannot in general define Gibbs distributions of finite order.

(ii) Let $F(x): E_{x} \mapsto E_{x}$ be an invertible linear map for all $x$, and let $x \mapsto F(x)$ be of class $C^{\mathrm{r}}$. If we define $\tilde{\varphi}(x)=F(f x)^{-1} \varphi(x) F(x)$, then $F \mathscr{L}_{\tilde{\varphi}}=\mathscr{L}_{\varphi} F, F^{-1} \mathscr{L}_{\tilde{\varphi}}^{*}=$ $\mathscr{L}_{\varphi}^{*} F^{-1}$. In particular, the Gibbs distributions for $\tilde{\varphi}$ are the same as the Gibbs distributions for $\varphi$.

To prove (ii) note that if $\tilde{\Phi}=F^{-1} \Phi$ and $\tilde{\Psi}=F \cdot \Psi$ we have $(\tilde{\Phi}, \tilde{\Psi})(A)=$ $\tilde{\Psi}(A \tilde{\Phi})=\Psi\left(F A F^{-1} \Phi\right)=\Psi(A \Phi)=\Phi \cdot \Psi(A)$, provided $A$ is a scalar, i.e. $A \in \mathscr{C}_{\mathbb{C}}^{\mathrm{r}}(U)$.

\section{The Zeta Function}

For every integer $m \geqq 0$ we write

$$
\zeta_{m}=\sum_{x \in \mathrm{Fix} f^{m}} \operatorname{Tr} \varphi\left(f^{m-1} x\right) \cdots \varphi(f x) \varphi(x)
$$

where $\operatorname{Tr}$ is the trace of operators in $E_{x}$. The zeta function associated with $\varphi$ is then defined by the following formal power series in $z$ :

$$
\zeta(z)=\exp \sum_{m=1}^{\infty} \frac{1}{m} \zeta_{m} z^{m}
$$


[It is not hard to see that this series converges for $|z| \exp P(\log |\varphi|)<1$.]

6.1 Theorem. Let $\mathbf{r}=(0, \alpha)$, i.e., let $\varphi$ be $\alpha$-Hölder continuous. Then

$$
d(z)=\frac{1}{\zeta(x)}=\exp -\sum_{m=1}^{\infty} \frac{1}{m} \zeta_{m} z^{m}
$$

converges for $|z| \theta^{\alpha} \exp P(\log |\varphi|)<1$, and its zeros in this domain are the inverses $\left(\lambda_{j}\right)^{-1}$ of the eigenvalues of $\mathscr{L}_{\varphi}$, with the same multiplicities.

The symbolic dynamics version of this theorem has been proved by Haydn [10] when $\varphi=\exp A$, and $A$ is a complex-valued function. Earlier results in this direction had been obtained by Ruelle [20], and Pollicott [14]. A general proof of the theorem is given in Appendix A.

We discuss now the case where $f$ is differentiable (as usual, $f$ and $\varphi$ are assumed to be $C^{\mathrm{r}}$, the case $\mathbf{r}=(0, \alpha)$ is not excluded).

6.2 Proposition. Let $f$ be differentiable and, for $0 \leqq \ell \leqq \operatorname{dim} M$, define

$$
\zeta_{m}^{\ell}=\sum_{x \in \mathrm{Fix} f^{m}} \frac{\operatorname{tr}_{\ell} \Lambda^{\ell}\left(T_{x}^{*} f^{-m}\right)}{\operatorname{det}\left(1-\left(T_{x}^{*} f^{-m}\right)\right)} \cdot \operatorname{Tr} \varphi\left(f^{m-1} x\right) \cdots \varphi(f x) \varphi(x),
$$

where $\operatorname{tr}_{\ell}$ is the trace of operators in $\Lambda^{\ell}\left(T_{x}^{*} M\right)$. Introduce the following formal power series in $z$ :

$$
d^{\ell}(z \varphi)=\exp -\sum_{m=1}^{\infty} \frac{1}{m} \zeta_{m}^{\ell} z^{m}
$$

Then

$$
\zeta(z)=\prod_{\ell=0}^{\operatorname{dim} M}\left[d^{\ell}(z \varphi)\right]^{(-1)^{\ell+1}} .
$$

This is because $\zeta_{m}=\sum_{\ell}(-1)^{\ell} \zeta_{m}^{\ell}$.

The interest of the above proposition is that $d^{t}$ may be viewed as a kind of Fredholm determinant of $1-z \mathscr{L}^{(\ell)}$ (see Corollary 3.5), as the following conjecture indicates.

6.3 Conjecture $A_{0}$. If $f$ is differentiable, then $d^{0}(z \varphi)$ converges for

$$
|z| \theta^{|\mathbf{r}|} \exp P(\log |\varphi|)<1 .
$$

The zeros of $d^{0}(z \varphi)$ in (6.1) are the inverse $\left(\lambda_{j}^{0}\right)^{-1}$ of the eigenvalues of $\mathscr{L}=\mathscr{L}^{(0)}$, with the same multiplicities.

Note that if we replace the bundle $E$ by $E \otimes \Lambda^{\ell}\left(T^{*} M\right)$ and $\varphi$ by $\varphi \otimes \Lambda^{\ell}\left(T^{*} f^{-1}\right)$, we obtain the following statement.

6.4 Conjecture $A_{\ell}$. If $\mathbf{r} \geqq 1$ (i.e., $\mathbf{r}=(r, \alpha)$ with $r \geqq 1$ ), then $d^{\ell}(z \varphi)$ converges for

$$
|z| \theta^{|\mathbf{r}|+\ell-1} \exp P(\log |\varphi|)<1 .
$$

The zeros of $d^{\ell}(z \varphi)$ in (6.2) are the inverses $\left(\lambda_{j}^{\ell}\right)^{-1}$ of the eigenvalues of $\mathscr{L}^{(\ell)}$, with the same multiplicities. 
We shall refer to $A_{0}$ and $A_{\ell}$ collectively as Conjecture $A$ (equivalent to $A_{0}$ ). In view of Proposition 6.2, we have

6.5 Consequence. If Conjecture $A$ holds, then $\zeta(z)$ is meromorphic in (6.1). i.e., for $|z|<\theta^{-|\mathbf{r}|} \exp -P(\log |\varphi|)$.

6.6 Comments on Conjecture A. Note first that Theorem 6.1 may be viewed as a Hölder version of Conjecture $A$. If $\mathbf{r}=(0, \alpha)$ and $f$ is differentiable, $\log d^{\ell}(z \varphi)$ converges for $|z| \theta \exp P(\log |\varphi|)<1$ when $\ell \geqq 1$. Theorem 6.1 and Proposition 6.2 then imply that $d^{0}(z \varphi)$ converges for $|z| \theta^{\alpha} \exp P(\log |\varphi|)<1$. In particular, this gives the $C^{1}$ version of Conjecture $A$.

Conjecture $A$ also holds in the $C^{\omega}$ case. [The $C^{\omega}$ case of Conjecture $B$ below was proved in [18], and we shall show (Proposition 7.3) that this implies Conjecture $A$.]

In the case of an expanding map $f$ of a compact manifold (i.e., $X=M$ ), and the trivial bundle $E=M \times \mathbb{C}$, Tangerman [27] has proved a weakened version of Conjecture $A$. Specifically, for integer $r$ and $\varphi=\exp A$, Tangerman shows that $\zeta(z)$ is meromorphic for

$$
|z|<\frac{1}{\operatorname{degree} f} \cdot \theta^{-r / 3 \operatorname{dim} M} \cdot \exp -P(\operatorname{Re} A),
$$

provided $r \geqq 6(\operatorname{dim} M)^{3}$. In particular, $\zeta(z)$ is meromorphic in $\mathbb{C}$ in the $C^{\infty}$ case.

6.7 Remark. In the $C^{\omega}$ case of Conjecture $A$, the Fredholm determinants $d^{\ell}(z \varphi)$ are entire functions of order 0 satisfying estimates

$$
\log \left|d^{\ell}(z \varphi)\right| \leqq\left[\log \left(||\left|\mathscr{L}^{(\ell)}\right||||z|\right)\right]^{\operatorname{dim} M+1}+\text { const., }
$$

where $\left\|\left|\mathscr{L}^{(\ell)} \|\right|\right.$ is some norm associated with the extension of $\mathscr{L}^{(\ell)}$ into the complex.

The above bound on the growth of $d^{\ell}$ is due to Fried [8], correcting an erroneous statement in Ruelle [18] (itself based on an error in Grothendieck [9]).

\section{Relating Conjecture A and Conjecture B}

The purpose of this section is to tie some loose ends, and provide proofs required in Sect. 6. We shall however argue more generally, allowing $\mathbf{r}$ to take all values.

For $k \geqq 0$ we define

$$
\begin{aligned}
\zeta_{m k}^{0}= & \sum_{\left(\vec{i}_{0}, \ldots, \vec{i}_{m-1}\right) \in I(k)^{m}} t_{\vec{i}_{0} \vec{i}_{1}} \cdots t_{\vec{i}_{m-1} \vec{i}_{0}} \\
& \cdot\left[\operatorname{det}\left(1-T_{x\left(\vec{i}_{0}, \ldots, \vec{i}_{m-1}\right)}^{*} f^{-m}\right)\right]^{-1} \\
& \cdot \operatorname{Tr} \varphi\left(f^{-m} x\left(\vec{i}_{0}, \ldots, \vec{i}_{m-1}\right)\right) \cdots \varphi\left(x\left(\vec{i}_{0}, \ldots, \vec{i}_{m-1}\right)\right) .
\end{aligned}
$$

From (7.1) and Proposition 2.4 we obtain

$$
\zeta_{m}^{0}=\sum_{k}(-1)^{k} \zeta_{m k}^{0}
$$

where $\zeta_{m}^{0}$ is defined in Proposition 6.2. Let us introduce the formal power series

$$
d_{k}^{0}(z \varphi)=\exp -\sum_{m=1}^{\infty} \frac{z^{m}}{m} \zeta_{m k}^{0} .
$$


7.1 Conjecture $B_{0}$. If $f$ is differentiable, $d_{0}^{0}(z \varphi)$ converges for

$$
|z| \theta^{|\mathbf{r}|} \exp P(\log |\varphi|)<1 .
$$

Its zeros in (7.4) are the inverses of the eigenvalues of $\mathscr{M}$, with the same multiplicity. In view of Remark 4.4 this implies the following consequence.

7.2 Conjecture $B_{k}$. If $f$ is differentiable, $d_{k}^{0}(z \varphi)$ converges in (7.4), and its zeros in (7.4) are the inverse of the eigenvalues of $\mathscr{M}_{k}$, with the same multiplicities.

We shall refer to $B_{0}$ and $B_{k}$ collectively as Conjecture $B$ (equivalent to $B_{0}$ ).

7.3 Proposition. Conjecture A follows from Conjecture $B$.

We shall prove this implication separately for each $\mathbf{r}$. In fact we shall only consider the case where $\mathbf{r}$ is finite, because the $C^{\infty}$ and $C^{\omega}$ cases then readily follow. Since Conjecture $B$ is known to hold in the $C^{\omega}$ case, Conjecture $A$ is a theorem in that case, as noted in Sect. 6.

In view of (7.2), (7.3) we have

$$
d^{0}(z \varphi)=\prod_{k \geqq 0}\left(d_{k}^{0}(z \varphi)\right)^{(-1)^{k}},
$$

where $d^{0}$ is defined in Proposition 6.2. If $|\lambda|>\theta^{|r|} \exp P(\log |\varphi|)$, let $m_{k}(\lambda)$ be the multiplicity of $\lambda$ as an eigenvalue of $\mathscr{M}_{k}$ (write $m_{k}(\lambda)=0$ if $\lambda$ is not an eigenvalue). Using Conjecture $B$ we see that Conjecture $A$ reduces to proving that the multiplicity $\bar{m}(\lambda)$ of $\lambda$ as an eigenvalue of $\mathscr{L}$ satisfies

$$
\bar{m}(\lambda)=\sum_{k \geqq 0}(-1)^{k} m_{k}(\lambda)
$$

The spaces $\mathscr{C}_{E, k}^{\mathrm{r}}$ introduced in Sect. 4.3 are mapped into each other by coboundary operators $\alpha_{k}$ defined in the usual manner:

$$
\mathscr{C}_{E, 0}^{\mathrm{r}} \stackrel{\alpha_{0}}{\longmapsto} \mathscr{C}_{E, 1}^{\mathrm{r}} \mapsto \cdots \mapsto \mathscr{C}_{E, k}^{\mathrm{r}} \stackrel{\alpha_{k}}{\longmapsto} \mathscr{C}_{E, k+1}^{\mathrm{r}} \mapsto \cdots .
$$

[The image by $\alpha_{k}$ of $\Phi \in \mathscr{C}_{E, k}^{\mathrm{r}}$ evaluated on $U_{i_{0}} \cap U_{i_{1}} \cap \cdots \cap U_{i_{k}} \cap U_{i_{k+1}}$ is the sum over $k+1$-terms $\left(i_{0}, \ldots, \hat{i}_{j}, \ldots, i_{k+1}\right)$ of the restriction to $U_{i_{0}} \cap \cdots \cap U_{i_{k+1}}$ of $\left.(-1)^{j} \Phi_{\left(i_{0}, \ldots, \hat{i}_{j}, \ldots, i_{k+1}\right)}.\right]$ The operator $\mathscr{M}_{k}$ acts on $\mathscr{C}_{E, k}^{\mathrm{r}}$, and it is readily checked that $\mathscr{M}_{k+1} \alpha_{k}=\alpha_{k} \mathscr{M}_{k}$.

It is possible to construct a smooth partition of unity $\left(\chi_{i}\right)$ associated with $\left(U_{i}\right)$. More precisely, there are $C^{\mathrm{r}}$ functions $\chi_{i}$ on $U$, with $\chi_{i} \geqq 0$, and support $\chi_{i} \subset U_{i}$ such that $\sum_{i} \chi_{i}=1$. [To construct the $\chi_{i}$ start with functions $\chi_{i}^{0} \geqq 0$ such that $\chi_{i}^{0}>0$ on $X_{i}$, and support $\chi_{i}^{0} \subset U_{i}$. Restricting $U$, as we may, to a smaller neighborhood of $X$, we assume that $\Sigma \chi_{i}^{0}>0$, and then obtain the $\chi_{i}$ by normalization.]

Using $\left(\chi_{i}\right)$, a standard argument shows that $\operatorname{im} \alpha_{k}=\operatorname{ker} \alpha_{k+1}$ for $k=0,1, \ldots$

We have therefore an exact sequence

$$
0 \rightarrow \operatorname{ker} \alpha_{0} \rightarrow \mathscr{C}_{E, 0}^{\mathrm{r}} \stackrel{\alpha_{0}}{\longrightarrow} \mathscr{C}_{E, 1}^{\mathrm{r}} \rightarrow \cdots \rightarrow \mathscr{C}_{E, k}^{\mathrm{r}} \stackrel{\alpha_{k}}{\rightarrow} \mathscr{C}_{E, k+1}^{\mathrm{r}} \rightarrow \cdots \rightarrow 0,
$$

where $\mathscr{C}_{E, k}^{\mathrm{r}}=0$ for sufficiently large $k$. For each $\lambda$ with $|\lambda|>\theta^{|\mathrm{r}|} \exp P(\log |\varphi|)$, let $P_{\lambda}$ (respectively $\left.P_{\lambda k}\right)$ be the residue of $(z-\mathscr{L})^{-1}$ (respectively $\left.\left(z-\mathscr{M}_{k}\right)^{-1}\right)$ at $\lambda$. This is a linear projection onto the generalized eigenspace of $\mathscr{L}$ (respectively $\mathscr{M}_{k}$ ) corresponding to $\lambda$; we have an exact sequence 


$$
0 \rightarrow \operatorname{im} P_{\lambda} \rightarrow \operatorname{im} P_{0 \lambda} \stackrel{\alpha_{0}}{\rightarrow} \operatorname{im} P_{1 \lambda} \rightarrow \cdots \rightarrow 0,
$$

and (7.5) immediately follows.

\section{Hyperbolic Julia Sets}

Our study of the zeta function was most conclusive in the $C^{\omega}$ situation. In particular, this includes the case of an invariant set $X$ for a holomorphic map $f$ of a complex analytic manifold $M$, when the conditions (a),(b),(c) of Sect. 1 are satisfied. If $f$ is a rational map of the Riemann sphere $M$, let $X$ be the Julia set (see for instance Brolin [6]). Then Conditions (b) and (c) hold, and (a) is expressed by saying that $X$ is a hyperbolic Julia set.

8.1 Corollary. If $X$ is a hyperbolic Julia set of the rational map $f$ of the Riemann sphere $M$, and $\varphi$ a real analytic function on $M$, the zeta function of Sect. 6 can be written

$$
\zeta(z)=\frac{d^{1}(z \varphi)}{d^{0}(z \varphi) d^{2}(z \varphi)},
$$

where $d^{0}, d^{1}, d^{2}$ are the Fredholm determinants of the transfer operators $\mathscr{L}^{\ell}$ on $\ell$-forms for $\ell=0,1,2$. They are entire functions of order 0 satisfying the estimates of Remark 6.7.

The above corollary makes more precise the results of [21]. Note that in [21], we studied the situation where $\varphi(x)=-\log \left\|T_{x} f\right\|$. It is of interest that one can estimate the order of the Gibbs distributions (which appear in resonances and as functional derivatives of the zeros and poles of $\zeta$ with respect to $\varphi$-see Sect. 5).

If $\varphi$ is holomorphic in a neighborhood of $X$ rather than just real analytic, (8.1) may be replaced by

$$
\zeta(z)=\frac{d_{H}^{1}(z \varphi)}{d_{H}^{0}(z \varphi)},
$$

where $d_{H}^{0}, d_{H}^{1}$ are Fredholm determinants of transfer operators acting on holomorphic functions and holomorphic 1 -forms respectively.

\section{Appendix A}

The purpose of this appendix is to give a proof of Theorem 6.1, or more precisely Theorem A.1 below. For definiteness we restate the general conditions of applicability of this result.

Let $X$ be a compact metric space, and $f: X \mapsto X$ a continuous map which is expanding, topologically mixing, ${ }^{7}$ and has local inverses. We assume thus

(a) there are $\varepsilon>0, \theta \in(0,1)$ such that

\footnotetext{
7 As indicated in Sect. 1, mixing is assumed to simplify the statement of results, but is not really needed
} 


$$
d(f x, f y) \geqq \theta^{-1} d(x, y)
$$

whenever $x, y \in X$ and $d(x, y) \leqq \varepsilon$.

(b) if $\mathcal{O}$ is a nonempty open subset of $X$, there is $n \geqq 0$ such that $f^{n} \mathcal{O}=X$.

(c) with $\varepsilon$ as above, if $x, y^{\prime} \in X$ and $d\left(f x, y^{\prime}\right) \leqq \varepsilon$ there is a unique $y \in X$ such that $f y=y^{\prime}$ and $d(x, y) \leqq \varepsilon$.

Let $0<\alpha \leqq 1$, and $E \mapsto X$ be a $d$-dimensional (real or complex) $\alpha$-Hölder bundle. [There is thus a finite open covering of $X$ trivializing $E$, and the transition functions are $\alpha$-Hölder $d \times d$ matrix-valued functions.]

A.1 Theorem. Let $\varphi: E \mapsto E$ be a bundle map over $f$ such that, for each local inverse $\psi$ of $f$, the map $\varphi^{\circ} \psi$ is $\alpha$-Hölder continuous.

Then, the formal power series

$$
d(z \varphi)=\exp -\sum_{m=1}^{\infty} \frac{z^{m}}{m} \sum_{x \in \text { Fix } f^{m}} \operatorname{Tr} \varphi\left(f^{m-1} x\right) \cdots \varphi(x),
$$

where $\operatorname{Tr}$ denotes the trace of operators in $E_{x}$, converges for

$$
|z| \theta^{\alpha} \exp P(\log |\varphi|)<1 .
$$

In this region, the zeros of $d$ are the inverses $\left(\lambda_{j}\right)^{-1}$ of the eigenvalues of $\mathscr{L}$, with the same multiplicity.

We may thus write

$$
(1-z \mathscr{L})^{-1}=\frac{\mathscr{N}(z)}{d(z \varphi)}
$$

where $\mathscr{N}$ is an an analytic operator-valued function defined in the domain (A.1).

As mentioned earlier, the symbolic dynamics version of Theorem A.1 was proved (for $d=1, \varphi=e^{A}, A$ Hölder) by Haydn [10), improving results of Ruelle [20] and Pollicott [14]. A theorem on 1-dimensional maps, based on Haydn's ideas has been obtained by Baladi and Keller [1].

A.2 Proof of the Theorem. Let $\left(X_{i}\right)$ be a Markov partition for $(X, f)$, and let us define

$$
\zeta_{m 0}=\sum_{i_{0}, \ldots, i_{m-1}} \operatorname{Tr} \varphi_{\left(i_{0}, i_{1}, \ldots, i_{m-1}, i_{0}\right)}\left(x\left(i_{0}, \ldots, i_{m-1}\right)\right),
$$

where the sum is over $m$-tuples such that $t_{i_{0} i_{1}}=\cdots=t_{i_{m-1} i_{0}}=1$ and $x\left(i_{0}, \ldots, i_{m-1}\right) \in$ Fix $f^{m}$ as defined in Sect. 2. Using (4.3) we have thus

$$
\zeta_{m 0}=\sum_{i_{0}, \ldots, i_{m-1}} \operatorname{Tr} \varphi\left(f^{m-1} x\left(i_{0}, \ldots, i_{m-1}\right)\right) \cdots \varphi\left(x\left(i_{0}, \ldots, i_{m-1}\right)\right) .
$$

The proof of Proposition 7.3 applies here also, and our problem is reduced to showing that

$$
d_{0}(z \varphi)=\exp -\sum_{m=1}^{\infty} \frac{z^{m}}{m} \zeta_{m 0}
$$

converges in (A.1), and that its zeros are the inverses $\left(\lambda_{j}\right)^{-1}$ of the eigenvalues of $\mathscr{M}$, with the same multiplicity. 
Since the Markov partition is arbitrarily fine, we may assume that it trivializes $E$. We may thus think of $\varphi$ simply as a function with values in the $d \times d$ matrices; $\operatorname{Tr}$ is the usual matrix trace.

We may write

$$
(\mathscr{M} \Phi)_{j}(x)=\sum_{i: t_{i j}=1} \varphi_{i j}(x) \Phi_{i}\left(\psi_{i j} x\right)
$$

where $\varphi_{i j}=\varphi^{\circ} \psi_{i j}$ is $\alpha$-Hölder continuous on $X_{j}$, and we know (see Sect. 4.7) that the essential spectral radius of $\mathscr{M}$ is $\leqq \theta^{\alpha} e^{P}$, where $P=P(\log |\varphi|)$. We now think of $\mathscr{M}$ as acting on the space $\bigoplus_{i} \mathscr{C}^{\alpha}\left(X_{i}, \mathbb{C}^{d}\right)$ of $\alpha$-Hölder functions $\Sigma X_{i} \mapsto \mathbb{C}^{d}$.

For $\varepsilon>0$, there are finitely many eigenvalues $\lambda_{j}$ of $\mathscr{M}$ such that $\left|\lambda_{j}\right| \geqq \theta^{\alpha} e^{P+\varepsilon}$. If $m_{j}$ is the multiplicity of $\lambda_{j}$ we may write

$$
\sum_{j} m_{j}\left(\lambda_{j}\right)^{m}=\sum_{j} \lambda_{j}^{m} \sum_{\gamma} \sigma_{j \gamma}\left(S_{j \gamma}\right)=\sum_{j \gamma} \sigma_{j \gamma}\left(\mathscr{M}^{m} S_{j \gamma}\right)
$$

where $\left(\sigma_{j \gamma}\right)$ and $\left(S_{j \gamma}\right)$ are dual bases of the generalized eigenspaces of $\mathscr{M}^{*}$ and $\mathscr{M}$ respectively to the eigenvalue $\lambda_{j}$.

For each allowed $m+1$-tuple $\left(i_{0}, \ldots, i_{m}\right)$, let

$$
X\left(i_{0}, \ldots, i_{m}\right)=\psi_{i_{0} i_{1}} \circ \cdots \circ \psi_{i_{m-1} i_{m}} X_{i_{m}} .
$$
There is an operator $Q^{(m)}\left(\right.$ as in Sect. 4.2) which identifies $\mathscr{C}^{\alpha}\left(\sum_{i} U_{i}, \mathbb{C}^{d}\right)$ with a
subspace of $\mathscr{C}^{\alpha}\left(\Sigma U\left(i_{0}, \ldots, i_{m}\right), \mathbb{C}^{d}\right)$, and we have

$$
\mathscr{M}^{m} S_{j \lambda}=\mathscr{M}^{(m)}\left(Q^{(m)} S_{j \lambda}\right)
$$

For each allowed $\mathbf{i}=\left(i_{0}, \ldots, i_{m}\right)$ we choose $\bar{x}(\mathbf{i}) \in X(\mathbf{i})$ and if $i_{m}=i_{0}$, we require $\bar{x}(\mathbf{i})=f^{m} \bar{x}(\mathbf{i})$, so that $\bar{x}(\mathbf{i})$ is uniquely fixed in that case, and equal to $x\left(i_{0}, \ldots, i_{m-1}\right)$.

As in the proof of Proposition 3.2, we shall use the Taylor expansion of $S_{j \lambda}$ at $\bar{x}(\mathbf{i})$, but we limit this expansion to the order $r=0$. With the same operators $K_{(m)}$ as in Sect. 4.7 we may thus write

$$
\sum_{j} m_{j}\left(\lambda_{j}\right)^{m}=\sum_{J \gamma} \sigma_{j \gamma}\left(\left(\mathscr{M}^{(m)}-K_{(m)}\right) Q^{(m)} S_{j \gamma}\right)+\sum_{j \gamma} \sigma_{j \gamma}\left(\mathscr{M}^{(m)}\left(c_{j \gamma}\right)\right),
$$

where $\left(c_{j \gamma}\right)$ has the constant value $S_{j \gamma}(\bar{x}(\mathbf{i}))$ on $X(\mathbf{i})$. For the first term in the right-hand side, (4.7) gives

$$
\left|\sum_{j y} \sigma_{j \gamma}\left(\left(\mathscr{M}^{(m)}-K_{(m)}\right) Q^{(m)} S_{j \gamma}\right)\right| \leqq \text { const. }\left(\theta^{\alpha} e^{P+\varepsilon}\right)^{m},
$$

where a polynomial in $m$ has been absorbed in the $e^{m \varepsilon}$ factor. If $\chi_{\mathbf{i} k}$ is the characteristic function (in $\left.\Sigma X\left(i_{0}, \ldots, i_{m}\right)\right)$ of $X(\mathbf{i})$ multiplied by the $k^{\text {th }}$ unit vector in $\mathbb{C}^{d}$, the second term of (A.2) may be written

$$
\sum_{j \gamma} \sigma_{j \gamma}\left(\mathscr{M}^{(m)}\left(c_{j \gamma}\right)\right)=\sum_{j \gamma} \sum_{k=1}^{D} \sum_{\mathbf{i}} S_{j \gamma k}(\bar{x}(\mathbf{i})) \sigma_{j \gamma}\left(\mathscr{M}^{(m)} \chi_{\mathbf{i} k}\right)=\sum_{\mathbf{i}} \sum_{k}\left[(1-\mathscr{P}) \mathscr{M}^{(m)} \chi_{\mathbf{i} k}\right]_{k}(\bar{x}(\mathbf{i})),
$$

where $\mathscr{P}$ is the projection corresponding to the part of the spectrum of $\mathscr{M}$ in $\left\{\lambda:|\lambda| \leqq \theta^{\alpha} e^{P+\varepsilon}\right\}$.

The right-hand side of (A.4) is the sum of two terms. The first is 


$$
\sum_{\mathbf{i}} \sum_{k}\left[\mathscr{M}^{(m)} \chi_{\mathrm{i} k}\right]_{k}(\bar{x}(\mathbf{i}))=\zeta_{m 0},
$$

because the sum over $\mathbf{i}$ contains only terms such that $i_{0}=i_{m}$.

There remains to study the second term of (A.4), i.e., up to sign

$$
\sum_{\mathbf{i}} \operatorname{Tr}\left[\mathscr{P} \mathscr{M}^{(m)} \chi_{\mathbf{i}}\right][\bar{x}(i)),
$$

where we have omitted the index $k$, and written $\operatorname{Tr}$ instead of $\Sigma_{k}$.

Keeping the definition of $\bar{x}\left(i_{0}, \ldots, i_{m}\right)$, we also choose $\bar{x}\left(i_{0}, \ldots, i_{\ell}\right) \in X\left(i_{0}, \ldots, i_{\ell}\right)$ for $\ell=0, \ldots, m-1$. We have then

and therefore

$$
\begin{aligned}
& \sum_{\left(i_{0}, \ldots, i_{m}\right)} \operatorname{Tr}\left[\mathscr{P} \mathscr{M}^{(m)} \chi_{\left(i_{0}, \ldots, i_{m}\right)}\right]\left(\bar{x}\left(i_{0}, \ldots, i_{\ell}\right)\right) \\
& \quad=\sum_{\left(i_{0}, \ldots, i_{\ell}\right)} \operatorname{Tr}\left[\mathscr{P} \mathscr{M}^{m-\ell} \mathscr{M}^{(\ell)} \chi_{\left(i_{0}, \ldots, i_{\ell}\right)}\right]\left(\bar{x}\left(i_{0}, \ldots, i_{\ell}\right)\right),
\end{aligned}
$$

$$
\begin{aligned}
\sum_{\mathbf{i}} & \operatorname{Tr}\left[\mathscr{P} \mathscr{M}^{(m)} \chi_{\mathbf{i}}\right](\bar{x}(\mathbf{i}))-\sum_{i_{0}} \operatorname{Tr}\left[\mathscr{P} \mathscr{M}^{m} \chi_{\left(i_{0}\right)}\right]\left(\bar{x}\left(i_{0}\right)\right) \\
= & \sum_{\ell=1}^{m} \sum_{\mathbf{i}} \operatorname{Tr}\left\{\left[\mathscr{P} \mathscr{M}^{(m)} \chi_{\mathbf{i}}\right]\left(\bar{x}\left(i_{0}, \ldots, i_{\ell}\right)\right)-\left[\mathscr{P} \mathscr{M}^{(m)} \chi_{\mathbf{i}}\right]\left(\bar{x}\left(i_{0}, \ldots, i_{\ell-1}\right)\right)\right\} \\
= & \sum_{\ell=1}^{m} \sum_{\left(i_{0}, \ldots, i_{\ell}\right)} \operatorname{Tr}\left\{\left[\mathscr{P} \mathscr{M}^{m-\ell} \mathscr{M}^{(\ell)} \chi_{\left(i_{0}, \ldots i i_{\ell}\right.}\right]\left(\bar{x}\left(i_{0}, \ldots, i_{\ell}\right)\right)\right. \\
& \left.-\left[\mathscr{P} \mathscr{M}^{m-\ell} \mathscr{M}^{(\ell)} \chi_{\left(i_{0}, \ldots, i_{\ell}\right)}\right]\left(\bar{x}\left(i_{0}, \ldots, i_{\ell-1}\right)\right)\right\} .
\end{aligned}
$$

We have thus

$$
\begin{aligned}
&\left|\sum_{i} \operatorname{Tr}\left[\mathscr{P} \mathscr{M}^{(m)} \chi_{\mathbf{i}}\right](\bar{x}(\mathbf{i}))\right| \\
& \leqq \text { const }\left\|\mathscr{P} \mathscr{M}^{m}\right\|+\text { const } \sum_{l=1}^{m}\left\|\mathscr{P} \mathscr{M}^{m-\ell}\right\| . \\
& \cdot \sum_{\left(i_{0}, \ldots, i_{\ell}\right)}\left\|\mathscr{M}^{(\ell)} \chi_{\left(i_{0}, \ldots, i_{\ell}\right)}\right\| \cdot\left[d\left(\bar{x}\left(i_{0}, \ldots, i_{\ell}\right), \bar{x}\left(i_{0}, \ldots, i_{\ell-1}\right)\right)\right]^{\alpha} \\
& \leqq \operatorname{const}\left[\left(\theta^{\alpha} e^{P+\varepsilon}\right)^{m}+\sum_{\ell=1}\left(\theta^{\alpha} e^{P+\varepsilon}\right)^{m-\ell}\left(e^{P+\varepsilon}\right)^{\ell} \theta^{\ell \alpha}\right] \\
& \leqq \text { const } \cdot m\left(\theta^{\alpha} e^{P+\varepsilon}\right)^{m} .
\end{aligned}
$$

From (A.2), (A.3), (A.4), (A.5), (A.6) we obtain finally

$$
\left|\sum_{j} m_{j}\left(\lambda_{j}\right)^{m}-\zeta_{m 0}\right| \leqq \text { const. } m\left(\theta^{\alpha} e^{P+\varepsilon}\right)^{m} .
$$

From this we see that

$$
\frac{d_{0}(z \varphi)}{\prod_{j}\left(1-\lambda_{j} z\right)^{m_{j}}}=\exp \sum_{m=1}^{\infty} \frac{z^{m}}{m}\left(\sum_{j} m_{j} \lambda_{j}^{m}-\zeta_{m 0}\right)
$$

converges for $|z| \theta^{\alpha} e^{P+\varepsilon}<1$, and the theorem follows. 


\section{References}

1. Baladi, V., Keller, G.: Zeta functions and transfer operators for piecewise monotone transformations. Preprint

2. Bowen, R.: Markov partitions for Axiom A diffeomorphisms. Trans. Am. Math. Soc. 154, 377-397 (1971)

3. Bowen, R.: Equilibrium states and the ergodic theory of Anosov diffeomorphisms. Lecture Notes in Mathematics, vol. 470. Berlin, Heidelberg, New York: Springer 1975

4. Bowen, R.: On Axiom A Diffeomorphisms. CBMS Regional Conf. Series vol. 35, Providence, R.I.: Am. Math. Soc. 1978

5. Bowen, R., Ruelle, D.: The ergodic theory of Axiom A flows. Invent. Math. 29, 181-202 (1975)

6. Brolin, H.: Invariant sets under iteration of rational functions. Ark. Mat. 6, 103-144 (1965)

7. Coven, E. M., Reddy, W. L.: Positively expansive maps of compact manifolds. In: Global theory of dynamical systems. Lect. Notes in Mathematics, vol. 819, pp.96-110. Berlin, Heidelberg, New York: Springer 1980

8. Fried, D.: The zeta functions of Ruelle and Selberg I. Ann. Sci. E.N.S. 19, 491-517 (1986)

9. Grothendieck, A.: Produits tensoriels topologiques et espaces nucléaires. Mem. Am. Math. Soc. vol. 16. Providence, R.I., 1955

10. Haydn, N.: Meromorphic extension of the zeta function for Axiom A flows. Preprint

11. Manning, A.: Axiom A diffeomorphisms have rational zeta functions. Bull. Lond. Math. Soc. 3, 215-220 (1971)

12. Nussbaum, R. D.: The radius of the essential spectrum. Duke Math. J. 37, 473-478 (1970)

13. Parry, W., Pollicott, M.: An analogue of the prime number theorem for closed orbits of Axiom A flows. Ann. Math. 118, 573-591 (1983)

14. Pollicott, M.: A complex Ruelle-Perron-Frobenius theorem and two counterexamples. Ergod. Th. Dynam. Syst. 4, 135-146 (1984)

15. Pollicott, M.: Meromorphic extensions of generalized zeta functions. Invent. Math. 85, 147-164 (1986)

16. Pollicott, M.: The differential zeta function for Axiom A attractors. Preprint

17. Ruelle, D.: A measure associated with Axiom A attractors. Am. J. Math. 98, 619-654 (1976)

18. Ruelle, D.: Zeta-functions for expanding maps and Anosov flows. Invent. Math. 34, 231-242 (1976)

19. Ruelle, D.: Generalized zeta-functions for axiom A basic sets. Bull. Am. Math. Soc. 82, 153-156 (1976)

20. Ruelle, D.: Thermodynamic formalism. Encyclopedia of Math. and its Appl., vol. 5, Reading, Mass: Addison-Wesley 1978

21. Ruelle, D.: Repellers for real analytic maps. Ergod. Th. Dynam. Syst. 2, 99-107 (1982)

22. Ruelle, D.: One-dimensional Gibbs states and Axiom A diffeomorphisms. J. Differ. Geom. 25, 117-137 (1987)

23. Ruelle, D.: Elements of differentiable dynamics and bifurcation theory. Boston: Academic Press 1989

24. Sinai, Ya. G.: Markov partitions and $C$-diffeomorphisms. Funkts. Analiz i ego Pril. 2, 64-89 (1968), English translation: Funct. Anal. Appl. 2, 61-82 (1968)

25. Sinai, Ya. G.: Construction of Markov partitions. Funkts. Analiz i ego Pril. 2, 70-80 (1968). English translation: Funct. Anal. Appl. 2, 245-253 (1968)

26. Sinai, Ya. G.: Gibbs measures in ergodic theory. Usp. Mat. Nauk 27, 21-64 (1972), English translation. Russ. Math. Surv. 27, 21-69 (1972)

27. Tangerman, F.: Meromorphic continuation of Ruelle zeta functions. Boston University thesis, 1986, (unpublished)

Communicated by J.-P. Eckmann

Received February 7, 1989 